\title{
Homophobie et structuration des jeux sportifs homosexuels
}

Yves Le Pogam, Philippe Liotard, Sylvain Ferez, Jean-Bernard Marie Moles et Guillemette Pouliquen

\section{(2) OpenEdition \\ 12 Journals}

Édition électronique

URL : http://journals.openedition.org/corpsetculture/823

DOI : 10.4000/corpsetculture.823

ISSN : $1777-5337$

Éditeur

Association Corps et Culture

Édition imprimée

Date de publication : 1 janvier 2004

ISSN : 1268-5631

Référence électronique

Yves Le Pogam, Philippe Liotard, Sylvain Ferez, Jean-Bernard Marie Moles et Guillemette Pouliquen, «Homophobie et structuration des jeux sportifs homosexuels », Corps et culture [En ligne], Numéro 6/7 | 2004, mis en ligne le 24 septembre 2007, consulté le 08 septembre 2020. URL : http:// journals.openedition.org/corpsetculture/823; DOI : https://doi.org/10.4000/corpsetculture.823

Ce document a été généré automatiquement le 8 septembre 2020.

(c) tous droits réservés 


\title{
Homophobie et structuration des jeux sportifs homosexuels
}

\author{
Yves Le Pogam, Philippe Liotard, Sylvain Ferez, Jean-Bernard Marie Moles \\ et Guillemette Pouliquen
}

1 Les recherches françaises en sociologie du sport ont été fécondes depuis les années 70 et montrent les différentes formes d'inégalités culturelles produites et reproduites par les sports, mais il est un champ qui est resté relativement silencieux contrairement aux travaux nord-américains florissants depuis les années 80 , celui des rapports entre le sport et l'homosexualité. Certes, l'homosexualité n'est pas ignorée de la recherche spécifique au sport, mais elle n'est pas toujours abordée comme un objet singulier et est intégrée, ou bien à une problématique plus générale relative au rapport masculin/ féminin dans le rugby (Saouter A., 2000), ou bien à un questionnement sur la souveraineté et sur la place des rites sportifs dans la générativité du social (Le Pogam Y., 1999a, 1999b), ou bien aux questions de l'intégration et de la déstigmatisation de minorités où figurent aussi les personnes handicapées (Marcellini A., Lefevre N., de Léséleuc E., Bui-Xuân G., 2000). Comme objet spécifique d'analyse, elle donne lieu à deux types d'interrogations: la place d'un événement sportif singulier comme l'organisation des Euro Games de Paris dans le mouvement sportif homosexuel (Falcetta L., 1997, Lefevre N., 1998) et la recherche des formes de manifestation de l'homophobie dans le sport (Pouliquen G., 2002 ; Liotard P., 2003). Quant aux recherches essentiellement nord-américaines, elles pourraient être rangées dans deux directions sans toutefois pouvoir être séparées par des frontières nettement définies : d'une part parce que leur champ s'applique aux sexualités hors normes et aux processus qui construisent les différenciations sexuelles dans les contextes historiques et culturels (Chamberland L., 1997 : 13) et d'autre part parce qu'elles ont aussi en commun d'être l'œuvre d'auteures "féministes» pour beaucoup d'entre elles. Elles se distinguent davantage par des principes épistémologiques qui les sous-tendent ${ }^{1}$. D'un côté, les Gay and Lesbian Studies sont centrées sur la prise en compte des variables sexuelles dans le sport et notamment l'emprise machiste imposée aux femmes ainsi que la présence de l'homophobie (Pronger B., 1990 ; Griffin P., 1992 ; Messner M.A., Sabo D.F. 1994 ; Kolnes J.-J, 1995), de l'autre, les Queer Studies, inspirées des théories de la déconstruction, 
questionnent le rapport homosexualité/hérérosexualité et remettent en cause les identités fixées, en partant du principe que la notion de déviance attribuée à la culture homosexuelle s'établit par rapport à la norme hétérosexuelle dominante jugée, elle, comme normalisée et naturalisée. Une des conséquences de ces approches consiste à chercher comment les identités se constituent par les pratiques culturelles de la vie quotidienne, dont le sport, et notamment la manière dont les homosexuels s'ajustent à l'expression hétérosexuelle comme dimension du genre et de la sexualité dans le sport de performance, soit en faisant abstraction de leur identité, soit en changeant les caractéristiques dominantes des rapports masculin/féminin (de Lauretis T., 1991 ; Stein A., Plummer K., 1996 ; Eng H., 1997).

2 Ces recherches s'orientent selon deux directions distinctes: la manifestation de l'homophobie dans le sport d'une part, et l'analyse des événements sportifs homosexuels d'autre part. Or, il semble qu'une attention particulière puisse être accordée à la relation entre l'homophobie et la structuration du sport homosexuel dont le développement depuis les années 80 fait qu'il peut être considéré comme un " fait social total » au sens maussien du terme (Mauss M., 1924). Il ne s'agit pas de rompre avec l'apport des recherches démontrant la présence de l'homophobie et/ou de la lesbophobie dans le sport « officiel », ni de nier le poids de la dominante masculine et hétérosexuelle. Le projet se propose plutôt de comprendre comment les sentiments d'injustice et de souffrance vécus par les homosexuel(le)s les engagent dans une forte mobilisation qui se concrétise par la mise en place d'organisations, de pratiques et d'événements sportifs autonomes visant à la fois la défense d'une cause et une aspiration à l'émancipation engendrant un mouvement social. L'intention est ici de lier les sentiments d'exclusion et de stigmatisation dont sont victimes les homosexuels dans le sport « officiel » à leurs capacités stratégiques à mobiliser leurs ressources pour structurer leurs organisations par une institutionnalisation afin d'obtenir des réponses face aux pouvoirs. Ceci ne va pas sans poser un certain nombre de questions.

3 Comment en effet comprendre l'existence de l'homophobie alors que les discours d'autorité des instances du sport «officiel» plaident pour l'inclusion de tous? Quelle est la force transgressive des jeux sportifs homosexuels par rapport aux valeurs sportives dominantes? Comment vivre la contradiction consistant à maintenir une préférence sexuelle et un style de vie singulier tout en collaborant - sur le mode réel ou imaginaire - avec les instances du sport « officiel» représentatives de l'ordre qui est combattu? Comment se vivent ces deux mondes que tout sépare et est-il possible pour les sportifs homosexuels de se fondre dans l'ordre dominant sans se confondre? À terme, cette volonté de reconnaissance d'une différence identitaire par le sport ne peut-elle pas conduire au renoncement de la subversion qui anime leur mouvement émancipateur? Ces questions appellent à l'analyse des jeux et des enjeux entre des phénomènes de production, ici, la créativité générée par l'affirmation d'un différentialisme fondé sur une préférence sexuelle minoritaire, et des phénomènes de reproduction, ici, le poids des conservatismes de la culture hétérosexuelle dominante dans les institutions sportives légitimées et légitimantes.

Homophobie et mobilisation des affects

4 Pour comprendre la créativité dont font preuve les homosexuel(le)s pour développer des formes sportives spécifiques, il est nécessaire de donner toute son importance à la culture des sentiments ou aux affects à condition de voir en eux, comme le suggère Pierre Ansart, des indicateurs d'action et de dynamique et non pas des épiphénomènes 
conjoncturels $(1979,1984,1988)$. Cela présuppose que l'imaginaire social constitutif de la culture homosexuelle, fait d'images identifiantes, de constructions du passé et d'anticipation sur une destinée qui s'écrit, est suffisamment cohérent pour se matérialiser dans des actions concrètes exprimant une revendication et renforçant les liens collectifs et les forces offensives d'un groupe stigmatisé. L'imaginaire social s'affirme par la production incessante de sens et de significations qui invitent à lire, non pas le passé, les permanences, les structures ou les situations sociales cristallisées, mais une société en train de se faire, en état de lutte et en création (Gurvitch G., 1950). Cette cohérence de l'imaginaire social peut s'objectiver dans la notion de mouvement social curieusement délaissée pour théoriser le sport homosexuel, mouvement constitué par la mobilisation des gays et des lesbiennes ${ }^{2}$ autour d'émotions, d'intérêts, d'espoirs générés par l'idée-affect d'injustice dont ils sont l'objet dans un univers où les représentations dominantes reposent essentiellement sur l'autorité de la norme hétérosexuelle ${ }^{3}$. Le sport n'est pas le reflet de la société, il fait système avec elle (Le Pogam Y., 1997). Il s'agit alors d'analyser les réactions provoquées par ce sentiment d'exclusion et d'apprécier son rôle comme catalyseur d'une lutte conduisant à l'autoorganisation d'un système sportif, comprenant des institutions, des organisations, des événements singuliers. Il s'agit en outre d'en saisir la portée sociale et la dynamique dans un mouvement social plus extensif intégrant des questions récurrentes comme la lutte contre le Sida, ou des questions plus récentes comme le mariage ou la parentalité des gais et des lesbiennes.

5 A l'origine de cette idée-affect d'injustice et d'incompréhension, se trouve une violence symbolique ${ }^{4}$ qui s'exprime par l'injure, la dépréciation, la raillerie à l'égard d'une préférence sexuelle autre, violence qui se repère aisément dans les discours et les pratiques homophobes. Les exemples abondent pour montrer une double voie de l'homophobie dans les institutions sportives "officielles", une violence proclamée mais aussi une violence fondatrice plus insidieuse qui s'inscrit dans la culture du silence (Liotard P., 2003). Ainsi, Pat Griffin (1992), discrimine des catégories dans les manifestations homophobes à l'égard des femmes sportives lesbiennes: le silence des organisations professionnelles qui refusent d'aborder cette question, le déni de la part d'entraîneurs, de directeurs et d'athlètes de la présence d'homosexuelles dans les sports en réponse aux inquiétudes des parents dans le cas où le silence est brisé, l'apologie de l'image féminine pour masquer sa préférence (vêtements, maquillage, sorties avec des hommes), et à côté de ces trois réactions défensives, de nouvelles stratégies lorsque le sport féminin est devenu plus visible et dépendant du marketing, comme la promotion d'une image hétérosexuelle des sportives dans les médias qui se distancie d'une image de la sportive lesbienne, les attaques contre les lesbiennes dans le sport de la part d'entraîneurs proclamant ouvertement leur politique lesbophobe et enfin, une préférence pour les entraîneurs masculins.

6 Les discriminations dont sont victimes les homosexuels au sein des institutions sportives officielles européennes sont répertoriées par l'European Gay and Lesbian Sport Federation, (E.G.S.L.F. ; 1999) dans un document où figurent des témoignages exprimant le pouvoir de l'ordre hétérosexuel, qui peuvent paraitre anecdotiques mais où se lisent des blessures affectant les usages du corps dans la vie quotidienne. Cela se manifeste par exemple au niveau des Fédérations officielles par le rejet des demandes d'adhésion de clubs gays ou lesbiens dont l'objectif explicite est de promouvoir leur émancipation et leur visibilité, au niveau des entraîneurs par l'exclusion des joueuses de football lesbiennes qui ne voulaient pas séparer leur vie privée de leur vie sportive ce qui 
amènera la dissolution de l'équipe afin que cet exemple soit suivi par d'autres entraîneurs, ou encore par le rejet de multiples joueuses de niveau international suite à l'aveu de leur homosexualité ou encore, de manière plus insidieuse, par le refus de nageurs adhérents à un club sportif officiel de s'entraîner avec des homosexuels d'un club parisien dans le même bassin.

7 Les recherches pionnières de Gert Hekma aux Pays-Bas portant sur 300 homosexuels des deux sexes dont la moitié habitent Amstersdam, montrent bien que la discrimination ne joue que si les homosexuels se déclarent comme tels ou s'ils font partie de clubs dont l'identité homosexuelle est affichée $(1994,1997)$. Le danger de se retrouver dans une atmosphère hostile conduit ces personnes stigmatisées à reculer leur coming out (sortie du placard), signe que la visibilité comporte des risques ${ }^{5}$. C'est bien ce dilemme que vit le sujet : révéler son homosexualité dans le sport mais risquer de subir des comportements homophobes, la taire, mais se hasarder à n'être jamais reconnu comme tel et vivre dans une clandestinité coûteuse au plan social, psychologique et culturel. Cette recherche montre que la grande majorité des homosexuels interrogés n'affichent pas leur préférence sexuelle et n'en parlent pas avec leurs camarades de sport, soit parce qu'ils ont peur, soit parce qu'ils considèrent que cela relève de la vie privée, alors que les hétérosexuels ne font aucun mystère de leurs relations amoureuses, de leur mariage, de leurs sorties avec les amis et échappent aux questions relatives à la sexualité (Hekma G., 1997).

8 Le sport, comme élément structurant de la vie quotidienne, exerce bien une fonction symbolique et pratique de séparation des préférences sexuelles, qui, dans ses formes silencieuses, exprime par l'indifférence blessante, l'imposition d'une culture hétérosexuelle dominante dont le pouvoir est d'autant plus fort qu'elle est incorporée et qu'elle se fonde sur l'impensable présence d'une sexualité « autre » dans le sport.

9 Cette violence homophobe n'affecte pas de manière équivalente les homosexualités gaies et lesbiennes dès lors qu'elles concernent les genres masculin/féminin. Les discriminations sont différentes selon qu'elles se rapportent aux hommes ou aux femmes, parce que, comme le montrent encore les recherches de Gert Hekma, les représentations se construisent sur des préjugés présents dans la société selon lesquels les gays sont efféminés et les lesbiennes masculinisées. C'est la raison pour laquelle beaucoup de jeunes gays s'accordant avec l'idéologie machiste du sport, estiment qu'il existe une incompatibilité entre leur homosexualité et le fait d'avoir un bon niveau sportiff. Ils quittent alors le sport et n'osent pas affirmer leur homosexualité. Les lesbiennes sont plus actives et plus visibles parce qu'elles sont pensées comme masculines et s'accordent alors avec l'idéologie dominante et les connotations qu'elle engendre. Elles s'affirment en priorité dans les sports collectifs, comme le football pratique où sont recensées par ailleurs le plus grand nombre de violences homophobes. Les hommes, après avoir rejoint un club gay et lesbien, délaissent plutôt les sports collectifs pour les sports individuels, sauf le volley-ball'.

10 Les travaux de J. Schuyf et L. Stoepler (1998) confirment que les raisons de l'adhésion à des clubs sportifs gays ou lesbiens sont d'ordre social : il s'agit de se sentir entre soi, d'être réellement soi-même dans ce temps libre, tout en conservant le souvenir malheureux de la pratique avec les hétérosexuel(le)s à cause de comportements machistes et de l'attention exacerbée à la recherche de la performance.

11 Ces quelques exemples suffisent pour comprendre l'importance des affects dans la dynamique instituante $\mathrm{du}$ sport homosexuel et dans l'appropriation d'un sport 
autonome gay et lesbien qui se structure sur une déconstruction des codes dominants du sport officiel. Cette mobilisation ne se constitue pas sur un désir spontané mais sur une réaction à l'homophobie directement proclamée ou plus insidieusement dispensée par les institutions du sport officiel qui renforcent les valeurs de virilité et de masculinité. L'autonomie a souvent été théorisée sur le plan individuel comme la capacité dont dispose la personne pour modifier l'effet des perturbations en provenance de son environnement (Barel Y., 1984). Elle peut aussi s'appliquer à un collectif, dès que les personnes s'agrègent autour de la défense d'une même cause et s'organisent en un mouvement pour faire face à une discrimination dont elles sont victimes dans la société et dans les institutions. Cette souveraineté devient un moyen de combattre les injustices en produisant un contre-pouvoir par une auto-organisation dynamisée par la puissance des affects et par le désir de réhabiliter des dimensions culturelles jugées tout à fait légitimes.

Il est alors possible de penser que cette souveraineté- qui s'affirme dans l'institutionnalisation du mouvement sportif homosexuel - vise à produire de nouvelles formes de symbolisations, rompant avec l'ordre sportif dominant. L'objectif consiste alors à conduire les communautés stigmatisées à affirmer une différence et à revendiquer des droits dont les membres se sentent privés. Dans cette perspective, le sport est perçu comme un excellent vecteur d'émancipation (Laberge S., 1995) visant à sauvegarder les différentialismes identitaires, précisément parce que les valeurs intégratives, rassemblantes et non discriminantes défendues par l'institution sportive officielle sont démenties dans la réalité des faits.

13 Les analyses historiques ou sociologiques portant sur la force du sport comme levier réactionnel des minorités culturelles à la domination - qu'il s'agisse de la lutte des femmes contre les institutions sportives misogynes, ou d'une volonté d'intégration des populations stigmatisées comme les sourds, les handicapés et les homosexuels (Marcellini A., Lefevre N., de Léséleuc E., Bui-Xuân G., 2000) -, tendent à montrer qu'il est possible de dégager au plan diachronique ce qui dans la sociologie des mouvements sociaux s'appelle des "répertoires d'actions collectives", c'est-à-dire des traits d'actions contestataires qui suivent un certain nombre d'étapes faisant passer les minorités de la marginalisation à la reconnaissance (Denis D., 1997). Ce passage est marqué par une phase d'autonomisation croissante du mouvement signifiée par la mise en place d'organisations et d'une institutionnalisation dont les formes visent à s'affranchir d'une phase précédente dans laquelle les rituels sociaux préexistants étaient détournés et l'horizon d'action élargi par l'appui de notables, pour inventer des formes d'actions originales et mettre en place des processus de pacification faisant reculer la violence (Tilly C., 1986, in Neveu E., 1996). Le peu de recul historique sur la structuration du mouvement sportif homosexuel qui émerge dans les années 80 , ne permet pas de discerner des scansions nettement repérables qui marqueraient le procès d'institutionnalisation. L'hypothèse peut être faite cependant d'une relation entre le degré de structuration des organisations, les liens de pacification avec le mouvement sportif officiel et leur ancienneté ou le degré de libéralisation des politiques nationales à l'égard de l'homosexualité ${ }^{8}$. Plutôt que de chercher des étapes définissant les invariants des processus de normalisation, il s'agit ici de scruter le brouillage dans les stratégies développées et les conflits d'où surgissent de l'inédit et des formes organisationnelles jamais stabilisées, tout en tenant compte de la complexité des liens entre les acteurs concernés. 
14 Il faut donc s'attendre à ce que se développe une stratégie paradoxale qui consiste tout à la fois pour le sport homosexuel à s'autonomiser et à se rapprocher des valeurs du sport officiel, celles du sport de performance. Une nouvelle hypothèse peut alors être avancée selon laquelle la structuration du sport homosexuel réactionnelle à l'homophobie se construit sur deux stratégies qui cohabitent en s'opposant : d'un côté, une stratégie d'autonomisation, différentialiste, séparatiste et souveraine dans laquelle le mouvement sportif homosexuel se gouverne selon ses propres lois; de l'autre, une stratégie de normalisation avec le sport officiel, agrégative et ensembliste où des échanges réciproques s'effectuent entre les types d'organisations ${ }^{9}$. Si la première permet un renforcement du "nous ", dans la seconde, la notion de «classe pour soi » semble perdre de son efficacité. Cette bipartition peut signifier que la mobilisation homosexuelle par le sport n'est pas unitaire et que la construction d'une image sportive homosexuelle est conflictuelle et son devenir incertain. Il s'agit bien de savoir comment s'écrit l'histoire de la confrontation/cohabitation de cette double logique et les paradoxes qu'elle comporte, signes de l'incertitude et de l'instabilité des cultures qui, en se rencontrant ou en s'ignorant, se font en se défaisant.

15 Ceci engage à ne pas simplifier les théories de la domination en n'y voyant qu'un registre d'actions passives qui présuppose l'assignation au statut de dominé, mais à l'envisager de manière relationnelle, c'est-à-dire en privilégiant les rapports de force entre deux types d'acteurs (Neveu E., 1996), ici des organisations sportives gaies et lesbiennes et des organisations sportives traditionnelles. Il convient alors d'analyser la dynamique des actions contestataires, leur codification dans un mouvement social qui s'institutionnalise, les processus qui lient et séparent les deux cultures antagonistes, bref les voies de la mobilisation collective guidées par un imaginaire social unifiant malgré les divergences d'action qui en résultent.

Méthodologie

16 Les deux stratégies (la production de formes originales et contestataires marquant une logique d'autonomisation et une prise de distance par rapport aux instances sportives officielles d'une part, et d'autre part les processus d'adhésion à la logique de performance des valeurs sportives "officielles»), seront recherchées à travers deux dimensions liées entre elles, présentées ici séparément pour des raisons de méthode.

17 D'abord, dans les idéologies fondatrices des institutions sportives homosexuelles émergentes (Fédérations, clubs, associations). Les idéologies peuvent en effet être comprises comme des ensembles symboliques en évolution à travers lesquels le groupe se définit, s'identifie, se met en scène, projette ses rêves et où s'investissent les pouvoirs, les craintes, les fantasmes et les angoisses (Ansart P., 1979). Ces idéologies sont politiques parce qu'en transformant l'imaginaire, elles conduisent à des conséquences symboliques et pratiques. Toute institution, en effet, se caractérise par des formes sociales de fonctionnement pratique s'appuyant sur des discours d'autorité qui symbolisent la spécificité des appartenances et servent de contrôle, de socialisation et de régulation, voire de contrainte à ses membres. Cette dimension idéologique des deux stratégies, différentialiste et agrégative, sera recherchée dans les objectifs des Fédérations ou associations sportives gaies et lesbiennes et dans la production des discours structurant l'institutionnalisation du sport homosexuel. Les indicateurs de la stratégie différentialiste retenus sont les termes majeurs renforçant la dimension persuasive de la formation d'une identité communautaire et souveraine. Ils mobilisent le «nous » (en soulignant leur exclusion, la solidarité), c'est-à-dire ce qui sert la cause 
homosexuelle. Les indicateurs de la stratégie agrégative sont les termes des discours marquant la volonté d'un rapprochement aux valeurs compétitives. Ils déclassent $a$ priori le primat de la revendication homosexuelle et signifient la normalisation par un discours plaidant pour l'intégration au sport de performance et à la compétition.

Après les discours vecteurs d'idéologie, c'est la force symbolique des événements sportifs homosexuels qui sera prise en compte, notamment la production de techniques du corps originales propres à dire la souveraineté, ou au contraire leur adéquation aux techniques sportives légitimées, signe de l'indépendance ou de la recherche d'une conformité avec les instances d'autorité du sport officiel. Il s'agit alors de faire valoir les formes sociales d'identification significatives de la culture sportive homosexuelle, dans la mise en scène des jeux sportifs organisés. Interroger ces événements organisés/ organisants, c'est montrer comment les rituels participent d'une volonté d'édifier ou bien une culture sportive originale, ou bien une culture proche de la performance et du milieu officiel. Les rites du sport homosexuel peuvent être compris comme «la mise en œuvre d'un dispositif symbolique qui construit des identités relatives à travers des altérités médiatrices » (Augé M., 1994, Le Pogam Y., 1999b). Cela peut se faire dans une volonté différentialiste en déconstruisant les codes dominants ou au contraire, dans un désir normalisateur, en les adoptant. Les indicateurs retenus seront les signes originaux constitués par les techniques du corps exprimées dans les gestuelles sportives produites à l'occasion des rencontres de tous niveaux, internationales ou nationales. Elles devraient confirmer cette puissance unitaire du message transmis à l'attention du dominant. Message qui l'interpelle dans le cadre d'une stratégie d'autonomisation en déconstruisant des gestuelles du sport officiel et en développant une socialité homosexuelle soudant une communauté, ou message qui vise à le séduire dans le cadre d'une stratégie de rapprochement, par l'adoption de ses règlements officiels, la multiplication des entraînements ou la recherche de la performance et des records. Le recueil des données touche à ces deux dimensions : idéologique et événementielle. Les idéologies - et les données organisationnelles dont elles sont issues - sont recueillies à partir d'un corpus comprenant des textes d'autorité produits par les instances du sport gay et lesbien, la Fédération des Gay Games (F.G.G) dont le siège est à San Francisco, l'European Gay and Lesbian Sport Federation (E.G.L.S.F.) créée en 1989 et établie à La Haye (Pays Bas) et le Comité Gay Paris Ile-de-France (C.G.P.I.F.) appelé actuellement la Fédération Sportive Gaie et Lesbienne C.G.P.I.F, créée à Paris en 1986. Ces trois instances, internationale, européenne et nationale, communiquent par des revues pour deux d'entre elles (Forum pour la F.G.G. et Sprint Express pour la Fédération Sportive Gaie et Lesbienne C.G.P.I.F) ou par divers documents relatifs aux discriminations homosexuelles dans le sport comme celui qui a été produit par L'E.G.L.S.F. Ces textes rendent compte de la vie des associations sportives. Ils contiennent des mots d'humeur, parlent des réformes statutaires, relatent des témoignages de sportifs à propos des manifestations auxquelles ils ont participé, de la création des nouvelles associations, des lieux d'entraînement, des relations entre les Fédérations. Bref, ils recèlent des données précieuses qui permettent de saisir la logique sur laquelle s'établit la force persuasive des discours associatifs malgré la difficulté de développement du mouvement sportif. Le corpus comprend aussi les statuts des trois types de Fédérations sportives gaies et lesbiennes composant l'échantillon, ainsi que les règlements qui fixent les adhésions et contractualisent les rapports entre les membres et leurs fonctions et enfin les activités les liant aux 
différentes instances du sport officiel. Ce sont là autant d'indicateurs permettant de saisir l'orientation politique des idéologies proclamées par les institutions sportives gaies et lesbiennes, et de voir, en acte, les pratiques concrètes mises en place au plan des structures organisationnelles pour dynamiser le mouvement sportif homosexuel. La dimension événementielle des jeux sera appréhendée, quant à elle, dans les manières de faire, dans les mises en scène des activités sportives et des spectacles à l'occasion des grandes manifestations internationales et nationales que sont les Gay Games (quasi Jeux Olympiques gays et lesbiens); les Euro Games, c'est-à-dire leur équivalent au niveau de l'Europe; les événements nationaux organisés par les Fédérations nationales. Certains événements précis feront l'objet d'une approche plus approfondie: les Gay Games de New-York en 1994 ou d'Amsterdam en 1998, les Euro Games de Paris en 1997, les manifestations européennes d'événements particuliers comme les tournois de natation organisés par le Paris Aquatique ou les tournois internationaux de volley-ball lesbien comme celui qui a été orchestré en 2002 par Contrepied, une association sportive gaie et lesbienne parisienne très active. Les rituels sportifs seront analysés d'après l'observation de documents vidéo des Gay Games de New-York (Ataman K., Brooks P. 1994) ou de tournois lesbiens de volley-ball (Good as you, 2002), ainsi qu'à partir de l'analyse iconographique de revues gaies et lesbiennes (Gai Pied, Têtu et Lesbia magazine qui consacrent aussi des articles aux jeux, et contiennent des témoignages de participant(e)s. Ce recueil est complété par l'analyse comparative des plaquettes des programmes des Gay Games depuis leur création en 1982, des Euro Games et des activités culturelles annexées à ces jeux et des tableaux des performances réalisées à cette occasion par les sportif(ve)s gays et lesbiennes ${ }^{10}$.

Ces données fondées sur l'observation, sur des discours, sur des documents fédéraux, se complètent par des séries d'entretiens non directifs réalisés auprès des responsables de l'organisation des Euro Games de Paris en 1997 (Président de la Fédération, Présidents d'associations de basket-ball, de natation, de badminton, de tennis et de football) et auprès de lesbiennes pratiquantes. L'objectif vise à faire émerger le vécu des pratiquants, leurs passions, leurs attentes, les enjeux, l'évolution du mouvement, les conflits, les attitudes vis-à-vis de la visibilité (ou de l'invisibilité), les rapprochements vers le sport la performance ou les liaisons avec le sport officiel. Enfin, un questionnaire a été administré aux pratiquant(e)s des Euro Games de Paris en 1997 (151 réponses), dans l'objectif d'identifier leur passé sportif, leurs motivations et leurs attitudes vis-à-vis du sport gay et lesbien et du sport officiel.

Les méthodes utilisées sont d'ordre qualitatif pour saisir les dimensions affectives par une analyse de contenu des discours (selon des catégories repérées à partir de la phrase, occurence des mots-clés), une grille d'observation des rituels (cérémonie d'ouverture des Gay Games, cérémonie de clôture et de remise des médailles, déroulement des activités et comportements des pratiquants, place du corps revendicatif, système d'entraide). Les méthodes sont également quantitatives pour compléter les données sur les rapports entre le sport officiel et le sport homosexuel et saisir les motivations des pratiquant(e)s.

24 La pluralité des moyens méthodologiques converge vers un seul objectif: saisir la double polarité stratégique dans laquelle s'écrit l'histoire de la structuration du mouvement sportif homosexuel vis-à-vis du sport hétérosexuel dominant : une logique différentialiste et une logique agrégative.

Une stratégie différentialiste d'affirmation 

de processus permettant au groupe contestataire de signifier une distance par rapport à la norme existante - le sport officiel et hétérosexuel -, par l'institutionnalisation d'un mouvement d'où émergent des Fédérations. Ces dernières, dans les discours qu'elles promeuvent et les pratiques qu'elles organisent, indiquent des directions d'actions. Quelles sont les formes repérables attestant d'une stratégie différentialiste et souveraine?

Des idéologies séparatistes des Fédérations internationales et nationales spécifiques, produisant des discours où la préférence sexuelle devient une cause à défendre contre l'homophobie. Ces discours font autorité car l'idéologie transmise n'est jamais neutre, elle sert de guide d'action à l'ensemble des membres. Elle se construit sur deux principes: la défense de la composante homosexuelle et l'élargissement du conflit à d'autres minorités qui souffrent, elles aussi, d'exclusion. C'est là un plaidoyer humaniste surplombant la seule référence à l'affirmation de l'homosexualité par les sports dont l'accès est ouvert à tous. L'idéologie promeut la construction d'une société sportive gaie et lesbienne ouverte dans laquelle la préférence sexuelle est clairement revendiquée ou seulement suggérée et qui s'inscrit dans la problématique plus extensive des droits de l'homme.

Une politique militante ouverte d'inclusion et de visibilité

Les Gay Games sont l'occasion pour la Fédération des Gay Games (F.G.G.) de réaffirmer les valeurs fondatrices du sport homosexuel qui doit se développer « En dehors de toute orientation sexuelle, de genre, de race, de religion, de nationalité, d'origine ethnique, de croyances politiques, d'habiletés physiques, d'habiletés artistiques ou athlétiques ou de statut HIV. Participer est réellement plus important que gagner, ainsi, les athlètes de tout âge et de tout niveau sont invités à se joindre à nous " (Plaquette des Gay Games d'Amsterdam, 1998). Cet objectif vise à assurer «le succès des jeux en maintenant les valeurs d'inclusion, de participation et de réalisation du meilleur de soi ». Ils permettent de "réunir tous les quatre ans la communauté gaie et lesbienne dans une grande célébration de fierté et d'unité » (ibid.), et participent à "l'amitié à travers la culture et les sports" (Slogan des Gay Games d'Amsterdam, 1998).

Cette politique se colore d'un aspect plus militant quant il s'agit de dévoiler l'homophobie et de plaider pour une visibilité. Ainsi, il s'agit pour l'European Gay and Lesbian Sport Federation (E.G.L.S.F.) de : «Lutter contre les discriminations basées sur la préférence sexuelle dans le sport, favoriser l'intégration et l'émancipation des gays et des lesbiennes, servir de support à leur coming out, échanger des informations et établir une coordination entre les groupes sportifs européens, fonder de nouveaux droits pour les gays et les lesbiennes, organiser des événements sportifs où les gays et les lesbiennes peuvent s'exprimer librement sans cacher leur préférence sexuelle». Ce principe de non discrimination figure dans l'article 1 des statuts de la Fédération Sportive Gaie et Lesbienne C.G.P.I.F. de Paris : «Elle (la Fédération) a pour objet de promouvoir les activités sportives, la lutte contre les discriminations liées à l'orientation sexuelle et la reconnaissance des droits des personnes lesbiennes ou gaies ». Son rôle est explicitement spécifié : «La Fédération sportive gaie et lesbienne CGPIF joue sa vocation citoyenne: être une force militante. Elle doit participer à la défense des droits des lesbiennes et des gays et à la lutte contre les discriminations liées à l'orientation sexuelle ». L'idée d'égalité et de visibilité qu'autorise le sport est reprise par le Président de la Fédération: «C'est au travers du sport que nous pouvons ensemble faire 
progresser les mentalités et assurer la visibilité non caricaturale des gays et lesbiennes. Dans une société encore homophobe, nous devons contribuer à sensibiliser le public, à nous confronter pacifiquement et au grand jour sur les terrains de sport. C'est ainsi que nous gagnerons le respect de tous et que nous contribuerons à l'égalité des droits de la personne, quelle que soit son orientation sexuelle » (Sprint Express, 14 mai 1999 : Editorial).

Il existe dans les mots-clés (orientation sexuelle, inclusion, participation, communauté, célébration, émancipation, égalité des droits, non discrimination, respect, visibilité...) une volonté politique d'attirer l'attention sur la notion d'homophobie et d'exclusion. Cela constitue aussi un appel revendicatif à user de l'image positive du sport comme un moyen pour inverser le processus de dénégation de l'orientation homosexuelle et pour parvenir à une équité sociale. Il s'agit bien d'un appel à la mobilisation construit sur l'injustice dans une société démocratique.

La généralisation aux droits de l'homme : l'extension de la cause

Le discours persuasif entend en outre élargir le conflit contre l'homophobie en appelant à une solidarité internationale. L'objectif vise à sensibiliser les consciences individuelles et les décideurs politiques en montrant que les jeux sportifs homosexuels s'inscrivent dans la volonté d'intégrer les exclus du système sportif orthodoxe, c'est-àdire les autres minorités. Le sport homosexuel est présenté sous un double aspect. Il est à la fois un analyseur idéologique de l'ordre sportif hétérosexuel homophobe (en montrant ce qu'il ne fait pas) et un catalyseur exemplaire des contestations grâce à la puissance des événements qu'il organise. La puissance du mouvement s'oppose au pouvoir de la norme dominante. Ceci est particulièrement visible dans deux indicateurs visant à comprendre l'internationalisation de l'exclusion: la lutte contre le Sida et le désir de sensibiliser des organisations internationales à l'exclusion à travers la notion de droits de l'homme.

31 Les événements sportifs sont en effet une occasion de parler du Sida certes, mais en n'en faisant pas un problème qui toucherait exclusivement les homosexuels car il concerne la politique de l'ensemble des Etats-Nations concernés. Ainsi, dans le contexte des années 80-90 où l'épidémie comportait beaucoup d'inconnues quant à l'efficacité des thérapies et à l'engagement des responsables politiques de la santé, la lutte contre le Sida occupait une place importante dans le sport homosexuel et sa ritualisation amplifiait symboliquement sa dramatisation. Par exemple, lors de la cérémonie d'ouverture des Gay Games de Vancouver en 1990, un coureur (Brent Nicholson) qui avait couru 10000 miles en 23 mois (de 1986 à 1987) pour informer les américains sur le Sida, portait à cette occasion le drapeau arc-en-ciel, symbole de la fierté gaie. Il a rappelé qu'il courait entre autres, en l'honneur de Tom Waddell (médecin, champion aux Jeux Olympiques, créateur des Gay Games en 1982 et qui meurt du Sida en 1987). L'observation des Gay Games de New York en 1994, confirme bien la présence d'athlètes sidéens dans l'activité de body-building notamment, sans que cela n'incommode qui que ce soit. Les sidéens présents sont fiers d'être là et de lutter pour la préservation d'un corps appelé à se dégrader sous l'effet du virus, dans une institution où la norme est celle du corps vaillant et performant. Il existe aussi pendant la durée des jeux, mais en dehors des stades, des manifestations périphériques très ritualisées dans les cathédrales où les chœurs invitent à chanter "We are living with Aids", auxquels se joignent les parents des gays ou des lesbiennes qui se battent pour leur dignité, dont certains ont perdu leurs enfants à cause du Sida (Gay Games de San Francisco, 1986). La révélation de sa séroposivité dans le sport pose des questions éthiques aux Fédérations 
sportives gaies et lesbiennes et est l'objet de débats démocratiques entre les responsables qui estiment que ce n'est pas là leur rôle, sans pour autant évacuer la relation sport-prévention-santét ${ }^{11}$.

Cette lutte contre le Sida se lie à la défense d'autres causes internationales comme celles des minorités ethniques, par exemple les Aborigènes lors des Gay Games de Sydney en 2002 ou les handicapés. La lutte homosexuelle ne relève pas exclusivement d'un communautarisme protectionniste, mais elle s'inscrit dans le registre plus extensif des discriminations dont sont victimes les minorités qu'elle inclut dans un tout. Ainsi, s'affiche le message d'une défense des "droits de l'homme" et d'une "solidarité internationale ». Ceci occupe une place importante dans les programmes des Gay Games qui, contrairement aux actuels Jeux Olympiques, ne se circonscrivent pas aux seules prestations physiques, mais s'accompagnent d'un programme culturel comprenant certes des conférences sur l'homosexualité mais aussi des événements créant des liens avec des organisations internationales sensibles à la défense des droits de l'homme (comme avec Amnesty International ou avec des représentants de Ministères de pays étrangers) bref, quelques 300 membres (Namibie, Afrique du Sud, Mexique, Inde...) lors des jeux de Sydney par exemple. Ici, il s'agit de regrouper les minorités et de viser la reconquête de droits en dénonçant les discriminations et en légitimant les liens avec la culture originelle, les identités qu'il faut faire resurgir et rendre visibles au regard de tous.

Dans les contenus de ces discours se manifeste l'affirmation d'une idéologie différentialiste visant la production d'images mobilisatrices fortes, constitutives d'une conviction militante et combative contre les forces conservatrices renforçant l'hétérosexualité et la marginalisation des minorités. Cela s'objective dans une orientation symbolique marquant la résistance à des idées et pratiques homophobes et ethnocidaires, en voulant donner vie à des cultures que les pouvoirs confinent à l'invisibilité et au silence. En cela, ces idéologies sportives sont politiques et normatives: comme schèmes collectifs d'interprétation, elles indiquent une voie à suivre qu'elles légitiment. Elles lient une rationalité, celle de la conquête de droits de la personne, à des sujets agissants qui se mobilisent contre la domination. Elles prennent tout leur sens en entrant en conflit avec les discours et les pouvoirs des institutions portées par une idéologie de maintenance qu'elles entendent provoquer. Cela se perçoit dans les couples binaires dont les oppositions symboliques sont constituées : visibilité/ invisibilité, respect des droits de l'homme/discrimination, inclusion/marginalité, solidarité/atomisation sociale. Cette dualité marque la puissance instituante des idéologies pour légitimer une lutte dont l'efficacité est liée à l'organisation d'un système d'associations et de Fédérations autonomes par rapport au système sportif institué.

34 La dynamique instituante de ce système d'organisations ne sera pas développée ici. Il faut mentionner cependant qu'elle renforce le primat idéologique des institutions gaies et lesbiennes et témoigne d'une stratégie souverainiste. Cette organisation faite de coopérations, d'assemblées, de conseils d'administrations, de rapports transcrits et transmis, de liaisons multiples et complexes en réseau, exprime malgré une bureaucratisation, une démocratie en acte, au sens de libre discussion et de confrontation des idées (puisque les clubs sont représentés lors des Assemblées Générales ce qui permet d'intégrer les conflits), dont la cohésion repose sur la défense identitaire homosexuelle, qui sert de référence symbolique conduisant à une auto- 
organisation. Cette libre gouvernance organisationnelle se démarque ici des fédérations sportives officielles et l'identité homosexuelle s'auto-produit en produisant le sport homosexuel sans qu'il soit nécessaire, ici au moins, d'établir des ponts avec le mouvement sportif orthodoxe dont la présence n'est pas ignorée. Cela sert à construire une image positive née de la capacité à créer des organisations suffisamment bien structurées pour contribuer à l'essor du mouvement gay et lesbien. A terme, ce développement autonome devrait parvenir à sensibiliser les structures sportives officielles à leur valeur sportive et à l'intérêt présenté par les homosexuel(le)s pour le développement du sport en général.

La mise en scène des événements sportifs

35 Le second indicateur pour comprendre la logique visant à instituer un système sportif autonome réside dans la ritualisation du spectacle. Le décodage des signes originaux que sont les activités et les gestuelles sportives produites à l'occasion des rencontres sportives gaies et/ou lesbiennes devrait pouvoir renforcer l'hypothèse de cette puissance faisant cohérence autour de la spécificité du message qui doit être décrypté par les cultures autres et qui, simultanément, confère un sentiment très fort d'appartenance à ceux qui le produisent. Les jeux sportifs homosexuels peuvent être compris comme des rites, non pas seulement pour leur aspect cyclique (tous les quatre ans pour les Gay Games), mais parce que la mise en scène des techniques du corps codées et formalisées est significative d'une culture dont la résonance symbolique est d'autant plus forte qu'elle se lie au sens que leur attribuent les participants et à son écho sur la réalité sociale. Dans cette stratégie d'affirmation, la production de techniques du corps originales rompt avec celles de la logique sportive orthodoxe afin que naissent des images identifiantes agrégeant les homosexualités en une force collective communautaire transcendant les individualismes et les particularismes liés à la division des sexes, des genres ou des préférences sexuelles. Cela autorise la formation d'un « être-ensemble-homosexuel» construit sur l'opposition aux normes et valeurs transmises par le sport hétérosexuel.

Des pratiques d'inclusion : la bascule de la masculinité dominante

Les Gay Games peuvent être considérés comme le paradigme des jeux sportifs homosexuels dans la mesure où ils expriment le système axiologique constituant le socle d'une éthique à défendre. Ils sont le catalyseur de la mobilisation gaie et lesbienne par l'ampleur grandissante du nombre de participants, le vecteur de la visibilité homosexuelle et le producteur d'une culture originale par l'invention des formes de pratiques proposées et par les adaptations des règlements du sport officiel. Ce sont là autant de signes permettant d'échapper à l'emprise du sport officiel dont l'application stricte des règles priverait d'accès au sport bon nombre d'homosexuels.

Les Gay Games initiés en 1982 par Tom Waddell possèdent avec ce dernier l'image du mythique père fondateur dont la mémoire participe à la cohésion communautaire pour deux raisons au moins. D'abord, il est le héraut d'une conception d'un sport ouvert à tous en montrant qu'il n'existe aucune contradiction entre l'identité sportive et l'identité homosexuelle. Ensuite, par son décès à cause du Sida, il symbolise le martyr d'une maladie endémique engageant une lutte permanente que rappelle la minute de silence lors de l'ouverture des Gay Games de 1986 et le rituel du Patchwork associé à la journée mondiale contre le Sida chaque 1er décembre qui permet aux amis des sidéens décédés, une ouverture du deuil en humanisant la mort, en la vivant collectivement, ceci contre l'anonymat et la dépersonnalisation (Le Pogam Y., 1999b). Bien que les Gay 
Games soient structurés sur le modèle olympique en se produisant tous les quatre ans (San Francisco 1982 et 1986; Vancouver 1990; New-York 1994; Amsterdam 1998; Sydney 2002 et Montréal prévu en 2006), l'appellation première donnée par Tom Waddell «Jeux Olympiques Gay » n'a pu être maintenue ${ }^{12}$. Cela n'empêchera pas l'essor de l'événement, lisible dans le nombre de participants (1350 en 1982, 14175 en 1998), de sports (11 en 1982, 31 en 2002), d'épreuves (409 en 1998), de nations représentées (45 en 1994 à New York et 88 à Amsterdam en 1998), de salariés (62 pour Amsterdam), de bénévoles (3 042 en 1998), de spectateurs assistant aux cérémonies d'ouverture (500 à San Francisco en 1986, 17000 à Amsterdam, 35000 à Sydney) ou aux cérémonies de clôture (9 000 en 1986 et en 1998, 15000 en 2002) ou aux épreuves (200 000 à Amsterdam, soit 45000 personnes par jour).

38 Cette dynamique qui rompt avec une faible médiatisation, s'explique par la richesse $\mathrm{du}$ réseau associatif préparant cet événement en organisant en Europe par exemple, des Euro Games qui se déroulent les années où les Gay Games ne sont pas programmés (La Haye 1992, 1993; Berlin 1996; Paris 1997; Zurich 2000; Hanovre 2001; Copenhague prévu en 2003 et Munich en 2004). Ici aussi, les événements prennent de l'ampleur d'année en année (par exemple en 1997 à Paris, 2000 sportifs, 13 disciplines officielles et 6 en démonstration; à Zurich en 2000, 3000 participants et 20 disciplines). A cela, il conviendrait d'ajouter les manifestations européennes, internationales ou nationales organisées autour d'un sport (comme le volley-ball, la natation, le badminton, le football, la lutte...), dont certains sont très importants comme le Trophée Paris Aquatique en 1999 comptant 600 personnes et 11 pays, ou le Tournoi européen de volley-ball à Paris en 2002 qui comprenait 400 participantes lesbiennes et 46 équipes (dont 23 équipes allemandes et 10 hollandaises, les autres pays comportant entre une et trois équipes).

39 Au-delà de ces données quantitatives témoignant de la massification du phénomène, la stratégie d'autonomisation du mouvement sportif gay et lesbien s'affirme lors des Gay Games dans des adaptations des règlements du sport officiel afin que la pratique s'ouvre au plus grand nombre, quels que soient l'âge, le sexe ou le niveau d'habileté sportive, ceci dans le respect des préférences sexuelles. Les pratiquants sont certes arbitrés selon les règles fédérales internationales et une surveillance s'exerce à l'égard du dopage dans certaines disciplines, mais les manières de faire mettent en scène des procédures d'inclusion génératrices du lien social homosexuel concernant la place des femmes, les variations d'âge ou de niveaux de performance et évidemment les préférences sexuelles.

40 Ainsi la mixité, comprise comme la possibilité de constituer des équipes mélangeant les sexes, existe dans des sports collectifs comme le basket-ball ou le football ou dans le body-building et le bowling.

41 Les variations d'âge sont prises en considération et plusieurs activités comportent des épreuves fondées sur cette différenciation comme le cyclisme ou les distances de course des critériums diminuent en fonction de l'âge. Le marathon se court selon des catégories d'âge, la natation à Sydney est divisée en deux catégories (pré-masters 18-24 ans, masters 25-29 ans, 30-39 ans, 40-49 ans, 50-59 ans etc.). De même il existe des partitions d'âge dans des épreuves comme le body-building, la danse, les arts martiaux, la lutte.

42 Les niveaux de performance permettent de différencier en outre les participants : par exemple au tennis de table (récréatif, national, international), volley-ball (avancé, très 
avancé), badminton (haut niveau, quelques compétitions, loisir avec quelques compétitions, loisir avec petites compétitions), cyclisme (novice, sport, expert) etc.

Enfin, les préférences sexuelles s'objectivent dans le choix des partenaires (masculin ou féminin) dans des activités comme le bridge (Amsterdam), le patinage sur glace en couples (New York). Les différences raciales sont annihilées par le choix de partenaires de races différentes et des épreuves existent pour les handicapés (danse en fauteuil avec une personne valide par exemple).

L'originalité des Gay Games s'affirme dans cette distance prise par rapport aux institutions officielles en démultipliant les disciplines en autant d'épreuves articulées sur la prise en compte de variables généralement exclues du sport hétérosexuel (âge, sexe, préférence sexuelle, niveau de performance) et constituent par là une éthique démocratique intégrant aussi les hétérosexuels. La déconstruction du sport hétérosexuel s'opère moins par une rupture avec des techniques du corps qui restent codifiées que par un double processus de catégorisations. Les catégories adoptées permettent l'inclusion de tous en unissant dans les compétitions ce qui est séparé (sexes, âges, handicaps, etc.) et en séparant ce qui est uni (les niveaux de performance, méconnus dans la logique olympique puisque des présélections existent). Bref, elles ébranlent les symboles de la masculinité dominante et la recherche extatique de la performance en développant une politique ouverte d'inclusion.

Des pratiques de rébellion et de dérision

Cette dualité inclusion/exclusion s'affirme aussi dans une dimension où les affects de rébellion sont manifestes et où la dérision est employée comme lutte politique pour signifier les discriminations du monde sportif officiel ou pour renforcer les stéréotypes liés aux homosexuels afin de montrer au monde hétérosexuel qu'ils sont capables de rire d'eux-mêmes et qu'ils ne sont pas dupes du regard négatif de l'autre. C'est la défense de la préférence sexuelle qui est majorée et qui devient un enjeu face à l'homophobie institutionnelle et sociale. Le sport, comme les arts du spectacle, est un levier où le dominant peut s'observer dans la dérision de la mise en scène des corps homosexuels et comme tel sert de contre pouvoir critique à l'ordre symbolique de la domination. En usant parfois du grotesque et de la farce par le détournement des codes sportifs admis, toute la dimension anthropologique de la charge subversive apparaît à l'égard du dominant, et par une inversion des codes, le dominé, dans la caricature du statut qu'il exprime à travers les gestuelles, se joue du dominant en lui montrant ce qu'il n'est pas et en lui livrant une image de soi à laquelle il ne peut s'identifier. Il s'agit d'un jeu de miroir où tout est transfiguré : l'auto-dérision n'est pas seulement un processus de défense collective mais se lit comme une dynamique qui force l'interrogation sur le jugement dépréciatif de l'Autre.

L'observation des jeux sportifs homosexuels montre que la variété des formes d'expressions corporelles les plus ludiques masquent une symbolique bien plus profondément enfouie venant signifier ce qu'est la pensée hétérosexuelle. Les jeux sont l'occasion de multiplier les messages de victimisation et de sensibiliser les affects en les politisant. Ainsi, les patineurs sur glace en couples aux Gay Games de New York expliquent qu'ils ont abandonné le sport parce qu'ils se faisaient traiter de "tantouses" et qu'ils entendaient faire quelque chose de différent dans leur prestation. L'un des deux patineurs s'est fermé la bouche au moyen d'une croix de scotch noir et est vêtu d'un pantalon noir serré et d'un tee-shirt kaki et déclare : « La politique officielle pour les gays dans l'armée officielle, c'est ne rien demander et ne rien dire, et 
comme on patinait aux U.S.A. on a pensé que l'on pourrait incorporer cela dans notre numéro en se mettant du scotch sur la bouche. Beaucoup de grands médias nous ont demandé si nous étions amants? Cela ne nous a pas traversé la tête. On a chacun notre petit ami. " Ils terminent leur réalisation, d'un niveau technique moyen certes, mais dans une gestuelle harmonieuse et synchrone, en s'enlaçant et en s'embrassant sur la bouche, avant d'enlever le scotch qui la recouvrait, sous les applaudissements chaleureux des spectateurs.

Une inversion des codes hétérosexuels peut se lire aussi dans la créativité dont font preuve les nageurs en détournant des pratiques exclusivement féminines du sport officiel, comme la natation synchronisée. Les compétitions sont mixtes, parfois les prestations des hommes devancent celles des femmes et le niveau sportif atteint est tel que l'épreuve de natation synchronisée figure comme exhibition aux Gay Games de Sydney. Une dérision qui déconstruit les stéréotypes et qui provoque de l'étonnement de la part des spectateurs lors des galas ou des compétitions mais qui ne manque pas, encore une fois, de questionner le sport officiel dans les frontières qu'il établit entre le masculin et le féminin. La portée symbolique de cette innovation est très forte, même si au départ il existe bien une dérision (" un truc de folles, autrement ce n'est pas intéressant » comme le dit un nageur du Paris Aquatique), car les prestations des hommes sont de très haut niveau et déconstruisent encore le sport hétérosexuel dans les barrières établies en montrant qu'elles ne répondent à aucune réalité sociale, mais qu'elles relèvent d'un construit de l'institution sportive officielle. En cela, il s'agit d'une réelle subversion de l'ordre dominant et d'un acte profondément politique masqué par la parodie et l'amusement de départ.

48 La dérision peut être aussi très festive, parodiant la formation des couples faite d'un homme et d'une femme et les tenues vestimentaires du sport officiel, poursuivant alors l'exubérance des Gay Pride. Ainsi, lors des Gay Games de New York, l'un des patineurs sur glace en couple (composé de deux hommes, un blanc et un métis), est vêtu comme une femme (perruque blonde bouclée, retenue par un bandeau rouge et un grand nœud sur le front, short rouge, grande ceinture rouge entourant la taille laissant voir une poitrine velue, collant mauve), ailleurs un coureur de 200 mètres court en guêpière à dentelle noire et porte une perruque blonde, certains maillots de bain sont très fleuris, des joueurs de basket portent des jupettes en étoffe vichy et se livrent à un ballet autour du ballon devant un public médusé qui applaudit. Lors des Euro Games à Paris en 1997, les Frontrunners avaient préparé un programme d'animation avec lancer de sacs à mains, course de relais avec déguisements (Dragqueens, juges déguisés en policiers et officiels en baigneurs 1900, coureurs en Astérix, bébés Cadum, etc.).

L'humour dépasse la seule enceinte des stades et continue de s'afficher dans la rue et il est possible de lire sur les tee-shirt aux Gay Games de Vancouver « Nobody knows I'm Gay ", "Les placards sont faits pour les vêtements ", même si des inscriptions homophobes sur les murs témoignent de rejet de l'Autre.

Tout cela contribue à donner au sport homosexuel l'image d'une fête masquant une dimension symbolique et qui rappelle le rôle du carnaval et l'inversion des codes dominant/dominé qu'il permet. Ces pratiques de rébellion et de dérision diffèrent d'autres formes de travestissements sportifs dans leurs significations, comme le déguisement quasiment obligé dans certains marathons - comme en France celui du Médoc-, car si dans les deux cas le ludique vise une transfiguration des normes du sport ségrégatif et compétitif, ici la portée sociale est politique au sens où la transgression affecte un groupe avec un enjeu identitaire revendiquant et fabriquant un "nous » 
communautaire mobilisateur. Faire du sport un jeu où se mêle tout ce que l'officiel exclut, est un enjeu de la construction du lien social qui passe moins par la reproduction des techniques du corps que par la production de formes originales dont la ritualisation ne vise pas au maintien d'une idéologie défensive, mais procède d'un dispositif élargi dont la finalité vise à ce que, à long terme, le dominant transforme son regard sur la culture de l'Autre et donc sur les fondements qui constituent ses propres manières de penser, de sentir et d'agir.

L'être-ensemble-homosexuel : une éthique communautariste

51 Il s'agit maintenant de dévoiler la fascination et les affects produits par les événements et les spectacles sportifs en s'attachant au vécu des participants. Certes, les passions lors de jeux sportifs apparaissent souveraines : elles sont l'expression d'une préférence sexuelle qui peut s'affirmer ici et maintenant, mais elles demeurent attachées au monde objectif. Cette socialité émergente est vécue comme immorale du côté du sport officiel car y prédominent la solidarité, l'entraide et non l'individualisme de la performance ou l'asservissement à des nationalismes.

52 Le lien social apparaît de manière récurrente dans les discours des participants aux événements sportifs spécifiquement gays et lesbiens par la majoration des notions de communauté et d'émotions.

53 Les cérémonies d'ouverture des Gay Games sont ainsi des moments particulièrement " sensibles ", au sens donné par Pierre Sansot (1986), c'est-à-dire qui nous affectent et retentissent en nous, car s'y s'affirme une communauté émotionnelle. Elles sont très festives avec le défilé des athlètes par nations ou par villes, avec drapeaux et pancartes et pour les plus grandes représentations des tenues uniformes. Les applaudissements, la joie exprimée et les hymnes contribuent à créer une communitas que Victor Turner définissait comme "une aire de vie commune » (Turner V.W., 1969) et une grande solennité règne lors de la proclamation du serment des jeux par les athlètes. Aux Gay Games de San Francisco, l'identité homosexuelle se manifeste par un lâcher de ballons de couleur arc-en-ciel et à Amsterdam, une nacelle monte dans le ciel dans laquelle deux filles s'embrassent.

54 Cette charge émotionnelle célébrant la cause homosexuelle s'objective dans ces témoignages de participants aux Gay Games de manière directe ou implicite : "Les Gay Games restent les seuls jeux mondiaux qui respectent la devise de de Coubertin, l'essentiel c'est de participer. Il s'agit moins de battre l'adversaire en face de soi, que de prendre plaisir à pratiquer le même sport que lui. Construire plutôt que détruire. On est loin des guerres larvées des Jeux Olympiques. Ici, personne ne joue pour un pays, mais pour une cause: la cause homosexuelle (Joueur de volley-ball à Amsterdam); « Je sais à présent que les lesbiennes et les homosexuels représentent une force, une énergie extraordinaire, que nous sommes seuls responsables de notre destin, et qu'un rêve d'une semaine, c'est déjà mieux qu'une médiocrité annuelle » (Participante aux Jeux de San Francisco); «On sort de là avec l'impression que le monde homosexuel est une réalité énorme, heureuse, éclatante de vie » (Participante aux Jeux de Vancouver).

55 Cette valorisation de l'idée de "communauté " est directement exprimée par les participants : «Ce qu'on prouve dans ces jeux ? C'est que comme athlète, on peut se mesurer à un niveau mondial. On le fait de notre côté afin de créer un sentiment communautaire. Il y en a qui critiquent ces jeux. Même des gays pensent que ce serait bien qu'ils ne soient pas nécessaires. Moi, je trouve ça bien qu'ils soient nécessaires. J'aime le sentiment communautaire qui est en train de se développer. C'est si difficile pour les gays de se développer, et c'est très important 
parce qu'on partage tous quelque chose: un héritage culturel, une psychologie qui nous est propre »; " Les gens parlent de célébration du corps à propos des jeux, mais pour moi, c'est plus que cela. C'est une célébration de la communauté qui inclut tout le monde, des sidéens jusqu'aux adeptes de la gonflette"; "Ainsi, quand je suis tombé, ils ont continué à m'encourager. On ne trouve pas cela partout. On se sent proches les uns des autres, et ça c'est très bien. Tu sens que tu fais partie d'une communauté»; "Je suis. Ça veut dire que j'existe, que je fais partie de la société, partie d'un tout. Et de voir tous ces gens-là qui se sont donnés du mal pour venir ici, comme à de vraies Olympiades, à mon âge, j'aurais jamais cru que c'était possible. Alors, j'en suis !».

Le lien social communautaire qui s'élabore dans cette atmosphère festive, demeure constant pendant toute la durée des jeux et se traduit encore dans des mots-clés récurrents comme, "affirmer ", "convivialité ", "plaisir » rompant avec le sport hétérosexuel; "Tu n'es plus isolée. Tu es avec d'autres personnes qui partagent les mêmes problèmes que toi »; «En fait, ce mode de vie avec d'autres personnes dans le cadre du sport est une chose très importante pour moi, car il y a un but commun d'épanouissement et de développement personnel. Il y a beaucoup de convivialité, d'échanges »; "En pratiquant du sport ensemble et en côtoyant d'autres personnes homosexuelles, cela m'a permis de m'affirmer petit à petit en tant que lesbienne, d'abord dans les associations, puis progressivement dans mon entourage "; "Au départ, ce qui nous rassemble, c'est d'abord le sport. Il y a un plaisir qui est différent à participer ici dans une ambiance qui nous est particulière que de participer à un quelconque tournoi hétérosexuel que ce soit "; "Ma prestation est appréciée et - ô bonheur, je décroche la médaille d'or. L'émotion est forte. J'ai le cœur dilaté et les larmes aux yeux. Je suis plus heureux que sij'avais remporté un championnat officiel. Il n'y a aucune agressivité entre les compétiteurs: les encouragements et les félicitations sont sincères, chaleureux et vont droit au cœur » (Vainqueur dans un art martial à Amsterdam).

Ceci se confirme par les observations d'événements de moindre importance comme le tournoi européen de volley-ball lesbien à Paris où les filles des différentes nations sont heureuses de se retrouver, s'enlacent, s'embrassent, proposent des hébergements aux étrangères.

Assurément, les jeux sportifs homosexuels sont l'occasion d'affirmer une visibilité de sa préférence sexuelle en réaction au rejet d'institutions comme le sport hétérosexuel, la famille, l'armée ou la religion, ceci afin de réhabiliter une image de soi et construire une identité qui cesse de se vivre comme marginale.

Ainsi la pratique du sport homosexuel permet de retravailler le stigmate en donnant l'assurance d'une normalité que ne peut réaliser le sport hétérosexuel, notamment par rapport aux stéréotypes masculin/féminin : "Enfin, on peut être soi-même. La plupart des gens pensent que les gays sont des "folles" ou des travestis, et ça, c'est un autre côté des gays que le public devrait connaître»; "C'est génial. C'est quelque chose qu'on ne permet pas normalement, faire de la compétition. C'est rare qu'on nous reconnaisse comme athlètes, des mecs virils quoi ! »; "C'est une occasion pour moi d'avoir un contact avec ma masculinité, mais sans abandonner pour autant ma féminité. Je suis une femme, et ce que me permettent ces jeux, c'est de l'être pleinement, c'est-à-dire, une lesbienne, une mère, une grand-mère... »; "Cette compétition m'a permis d'être ce que j'appelle "normal". Sur le podium, au milieu des autres compétiteurs, des autres athlètes, on m'a jugé selon les mérites de mon travail, et cela, pour moi, ça a été super. C'est pas souvent que le monde me donne cette chance ».

60 Cette pratique communautaire sert de soutien contre les pressions familiales ou sociales, comme pour cette lutteuse noire aux Gay Games de New York dont les parents 
faisaient partie d'un groupe religieux et lui mentionnaient qu'un passage de la Bible lui rappelait qu'elle était possédée par le démon et qui déclare: "On m'a dit que Dieu ne m'aimait pas parce que j'étais une lesbienne. Je sais aujourd'hui que Dieu m'aime et je suis une lesbienne ", ou cette joueuse de basket-ball d'origine israélienne, entraîneuse d'un club lesbien et féministe qui confesse : «Ça veut dire beaucoup pour moi d'être ici. Je fais mon coming out et c'est très important qu'il s'agisse d'une compétition gaie et lesbienne. En raison de l'importance de la religion, il est difficile d'être out. Tant qu'on en parle pas, ça va »

Dans ce registre communautaire, l'individu se perçoit comme une personne car il est lié à un collectif en affirmant, comme le montrent les travaux de Michel Maffesoli « un être-ensemble» fondé sur l'émotion pensée comme une véritable structure anthropologique permettant de créer des formes de solidarité, plutôt qu'un « devoir être » (Maffesoli M., 1982, 1988, 1990, Le Pogam Y., 1998). Mais il est cependant possible, dans ce cas particulier, de se distancier de la thèse de l'auteur pour qui existe une «transfiguration du politique » (Maffesoli M., 1992) dans la mesure où la politique aurait fait son temps et le présentéisme aurait supplanté l'utopie des lendemains qui chantent. Ici au contraire, émotion et rébellion sont connectées car les sentiments éprouvés ne s'arrêtent pas en même temps que les jeux et représentent une force instituante qui se poursuit après eux pour amplifier le mouvement de mobilisation et transformer les codes culturels dominants dans l'espoir de rendre l'avenir meilleur.

La mise en scène des événements sportifs homosexuels permet de saisir la portée symbolique des techniques du corps qui suppose, comme le suggère Marc Augé, de ne pas analyser les significations du rite en le circonscrivant à l'espace restreint dans lequel les pratiques s'organisent - ici les stades, les gymnases, les piscines, etc.-, mais d'en apprécier les effets sur l'environnement psychologique, social et politique (Augé M., 1994). S'agissant de ces jeux, envisagés ici à la manière de l'idéal-type wébérien depuis une stratégie d'autonomisation, ils montrent que l'impact transformateur affecte les participants dans leurs dimensions psychiques (dans une affirmation identitaire), sociale (le lien social et le désir de transformer le regard de l'autre), et politique (en conscientisant tous les Etats-nations à l'exclusion, en ne se circonscrivant pas uniquement à la défense de l'homosexualité).

L'idéologie différentialiste des textes institutionnels du mouvement sportif homosexuel, la structuration de leurs organisations et la force symbolique des rituels mis en scène, témoignent d'une dynamique instituante souveraine dont la finalité est militante et politique, même si elle n'est pas toujours explicitement proclamée. Les indicateurs choisis ont permis de repérer une scansion avec le sport hétérosexuel, mais le développement du sport homosexuel ne saurait totalement s'autonomiser de l'ordre dominant. Toute autonomie, comme le pense Edgar Morin (1990) contient son contraire, c'est-à-dire la dépendance, deux notions qui ne doivent pas être pensées séparément, mais dialogiquement. C'est cette deuxième stratégie qu'il convient d'analyser maintenant.

Une stratégie agrégative de normalisation

Une des premières formes de métissage apparue dans le processus d'autonomisation du mouvement sportif homosexuel consistait dans «l'emprunt » des techniques sportives instituées mais en leur donnant un autre sens, afin de faire valoir la différence liée à la préférence sexuelle. Il s'agit maintenant de savoir s'il n'existe pas de liaisons plus profondes entre les deux types de cultures antagonistes et quelle stratégie est développée en direction du sport officiel, sans nécessairement qu'elle soit - comme 
pour la précédente -, le résultat d'actions uniquement rationnelles. Une position séparatiste, souveraine et radicale coupée de toute instance institutionnelle officielle légitimée et légitimante représentative du sport officiel est-elle louable pour la défense de la cause homosexuelle? Au-delà donc de l'importation des techniques sportives codées et formalisées, est-il possible, dans la structuration du mouvement sportif gay et lesbien, de voir émerger une autre ligne stratégique amplifiant paradoxalement les échanges avec le sport hétérosexuel comportant le risque éventuel de le conduire à une assimilation, fin extrême d'une logique de déstigmatisation?

Pour des facilités de comparaison, le système de preuves de cette stratégie agrégeant le sport homosexuel aux valeurs du sport dominant sera recherché dans les pratiques et les discours par des dimensions et des indicateurs identiques à ceux qui ont attesté les stratégies d'autonomisation. Cependant, l'observation des logiques structurelles sera approfondie, de manière à saisir la logique paradoxale qui anime les institutions du sport homosexuel.

Des idéologies ensemblistes

Dans la perspective normalisatrice, la participation au sport hétérosexuel est considérée comme un moyen de normalisation dans le sens où elle peut permettre l'intégration des homosexuels dans le sport officiel. Le sport comme fondement d'une cause, n'est plus seulement celui qui se développe en marge du mouvement sportif officiel, mais celui qui se lie aux institutions de l'ordre symbolique, afin d'éviter un narcissisme collectif communautaire et sortir d'un ghetto auto-satisfait de sa contestation. C'est bien ce propos qui a été au centre d'un colloque organisé pour le 30ème anniversaire de l'E.G.S.L.F., «Building bridges between regular and gay/lesbian sport » (1999) où, dans sa conférence introductive, le Président de la G.I.S.A.H. (qui est aussi co-Prédisent de l'E.G.S.L.F.) posait la question du métissage entre les deux types d'organisations en notant qu'il est évident que les organisations sportives officielles nationales et européennes ne sont pas prêtes à traverser le pont vers les gays et les lesbiennes dans la société et dans le sport en particulier. Il se posait les questions de la longueur du pont, de sa nécessité et de savoir s'il pouvait se traverser dans les deux sens.

Cette construction de passages vers l'institution légitime va s'appuyer sur la valorisation d'un discours intégratif de la part des institutions sportives homosexuelles et sur la valeur de la compétition pour parvenir à cette finalité. Ainsi, un article des statuts de l'E.G.S.L.F. mentionne bien cet objectif : « Combattre pour le fait que les gays et les lesbiennes aient une place respectable dans le sport régulier, compris comme un droit de l'homme»; "Promouvoir l'intégration et la reconnaissance mutuelle dans le sport entre les organisations sportives régulières et gaies et lesbiennes européennes ${ }^{13}$. Ce discours est relayé par certains présidents de clubs gays et/ou lesbiens : « Pour toutes les associations de sport gay et lesbien, il n'y aurait pas d'intérêt à pratiquer du sport sans la participation aux compétitions et sans l'intégration au sport hétérosexuel. Cela semble nécessaire à la vie du club sportif. C'est un objectif qui donne le sens et fixe la direction pour le club"; " Nous sommes à un point où nous devons choisir entre la pratique du sport juste pour le loisir et le sport officiellement reconnu. C'est une dimension qui doit être prise par tous les membres de l'E.G.S.L.F., probablement sport par sport » (Le Président d'un club de volley-ball gay et lesbien). Ces idéologies sont travaillées par des structures qui s'ouvrent au sport hétérosexuel.

Des structures intra-organisationnelles 
68 Malgré une volonté affichée de structurer le sport homosexuel sur le registre affinitaire, il existe de nombreux indicateurs qui témoignent de références au modèle officiel et dont l'effet engendre des modifications dans les structures internes en les orientant vers la recherche d'une plus grande lisibilité et d'une réelle professionnalisation.

69 Cela se perçoit dans la création envisagée pour 2004, de nouveaux événements sportifs proches du système dominant, comme les World Gay Winter Games (G.W.G.), quasi équivalents des Jeux Olympiques d'hiver qui s'inscrivent dans la suite logique de la création des Gay Games. Cela devrait aussi s'accompagner de l'émergence des European Winter Games, comme existent les Euro Games ou les Championnats européens dans le sport officiel. Mais, ici encore, l'éthique fondatrice demeure identique, car si les sports proposés sont ceux qui traditionnellement figurent lors des Jeux olympiques d'hiver, à leur différence, ils seront ouverts à tous, sans différenciations d'habiletés physiques avec la présence en annexes d'événements festifs et culturels.

70 Cette recherche de concordance s'effectue encore, de manière moins démonstrative mais tout autant significative, dans les réformes de statuts des organisations. L'objectif est de se doter d'une image sportive reconnue en adoptant alors les signes du système dominant. Ainsi la Fédération Sportive Gaie et Lesbienne C.G.P.I.F. a changé les noms de «Bureau» et de "Conseil d'Administration» en "Bureau Directeur» et "Comité directeur». Il s'agit de "se rapprocher des présentations des fédérations classiques et reconnues d'utilité publique ", en limitant le rôle du "Bureau Directeur » à la gestion, voire à la représentation de la Fédération, et celui du « Comité directeur » à la mise en œuvre de la stratégie adoptée par les Assemblées Générales (Sprint Express, 16, 1999).

71 Le Président de cette même Fédération, vise aussi à donner plus de professionnalisme à la structure afin de satisfaire l'objectif de "Mieux défendre l'image des homosexuels dans la société et promouvoir une autre idée du sport, conviviale et compétitive ». Cela nécessite de catalyser les ressources humaines ("Il nous faudra attirer, motiver, responsabiliser et légitimer davantage de volontaires bénévoles »), et de conduire, à l'image des grandes associations sportives obligées de se restructurer sous l'effet des contraintes environnementales (phénomènes de contingence structurelle), une segmentation efficace des tâches nouvelles, en départementalisant les fonctions en cinq équipes : " Ressources " pour gérer l'image de la Fédération (communication et marketing), " Relations extérieures" (liens avec les organisations homosexuelles et les Pouvoirs publics), «Relations Medias» (communication presse), «Equipe Réseau France» (aide au développement des associations sportives gaies et lesbiennes), "Equipe Sponsoring " (dossiers de parrainage, sponsors). A ces divisions, s'ajoutent cinq « Equipes projets » : Equipe «Marche homosexuelle» (Participation aux marches des fiertés homosexuelles), Equipe «Team France » (organisation de la délégation française pour les Gay Games et les Euro Games), Equipe "T.I.M. Paris» (coordination pour les tournois internationaux multisports), Equipe « Fêtes» (organisation des animations), Equipe « Fédération jeux gais » (avis de la Fédération dans le cadre de la Fédération des Gay Games).

Les structures inter-organisationnelles

72 Il semble que la communication entre les instances homosexuelles et les autorités sportives de tutelle soit d'autant plus dense que les nations connaissent une tradition sportive homosexuelle ancienne ou que les niveaux de performance atteints sont susceptibles d'être reconnus par les institutions sportives officielles. Les organisations sportives homosexuelles s'agrègent aux organisations culturelles sensibles à toute 
forme d'exclusion et aux instances du sport fédéral afin de développer le sport récréatif et le sport de compétition et parfaire leur visibilité.

Ainsi, il existe aux Pays-Bas depuis 1975, la Netherland Cultural Sport Federation (N.C.S.), une association multisports proposant 36 activités et 22500 sportifs en 1999, dont la vocation est d'ouvrir le club à toutes les minorités ethniques et culturelles et aux personnes présentant des handicaps, et ceci dans la double orientation du loisir et de la compétition. La N.C.S. coopère avec le Ministère et le Conseil Olympique (NOC.NSF) et une Charte de fair play et de tolérance (The Fair Play and Tolerance Charter) a été signée par les trois instances en 1997 à laquelle adhèrent 57 autres organisations. Ainsi, les structures sportives homosexuelles trouvent-elles un relais dans des organisations dont les finalités explicites visent à lutter contre les formes de discrimination, y compris celles qui touchent à la préférence sexuelle.

Cette communication inter-organisationnelle témoigne de l'ouverture du système des sports homosexuels. Elle se manifeste aussi en Italie où l'histoire du sport débute en 1989 par la création d'un club de natation (Gruppo Pesce Milano), qui passe très vite du niveau loisir à celui de la compétition et se lie aux Fédérations nationales et internationales de natation et au mouvement du sport pour tous italien (U.I.S.P.), ceci avec une pleine visibilité puisque les fondements de cette organisation s'appuient sur le rejet de toute attitude homophobe.

Les organisations sportives homosexuelles mettent très rapidement en œuvre des stratégies de développement en organisant des tournois mondiaux ou nationaux exclusivement gays et lesbiens, dès que l'activité a pris de l'ampleur, comme en natation ou en athlétisme, ceci dans des pays où le sport homosexuel a déjà une histoire (comme en Europe aux Pays-Bas ou en Allemagne). Cela permet d'intégrer dans la sphère sportive de compétition, les clubs émergents (comme en natation les Panteres Grogues, un club espagnol de Barcelone invité à rencontrer des nageurs du club plus confirmé qu'est le Paris Aquatique parisien). Mais les rencontres s'ouvrent aussi aux compétitions organisées par la Fédération de natation, avec l'enregistrement des records officiels.

76 Cette progression dans la normalisation fait penser à certains présidents de clubs que c'est là, la " voie royale ", à tel point qu'il est suggéré que le choix doit se faire, dans le cadre de la politique de l'E.G.S.L.F., entre le développement du sport loisir et le sport officiellement reconnu, entre les associations sportives sans licences et celles qui sont sous une autorité fédérale officielle (Gouyet S., 1999). Un club comme le Paris Aquatique devient le parangon de la réussite d'insertion sportive des gays dans le mouvement sportif officiel, dans la mesure où son autorité sportive lui a permis une reconnaissance de la Fédération Française de Natation qui a fourni les infrastructures et les ressources humaines pour assurer le succès d'événements mondiaux comme l'International Gay and Lesbian Aquatics (I.G.L.A) Championship de Paris, en 1999. Les nageurs du Paris Aquatique traduisent bien le changement de la pensée officielle : "Quand on est allé à la Fédération Française de Natation, au début, ils ont dit "Tiens! Les "pédés" ils viennent nager avec nous" et quand on a commencé à gagner des médailles c'est plus pareil». Ce caractère d'exemplarité doit servir de modèle aux autres clubs : "Mais qu'attendent les autres clubs pour lui emboitter le pas? Il suffit de retirer un dossier à la D.D.J.S. (Direction Départementale de la Jeunesse et des Sports), de le compléter de patienter quelques mois... Du doigté dans les relations humaines, un dossier solide, des entrainements passés au scanner d'enquêteurs sur place, plus un travail intelligent auprès du cabinet du Ministre au sein duquel on croit deviner 
quelques signes de sympathie à l'égard de notre cause, et l'affaire est jouée » (Sprint Express, 16, octobre 1999).

L'appel à la licence, symbole d'appartenance au réseau sportif officiel traduit encore cette stratégie de normalisation. Ainsi, un objectif de l'équipe « Réseau France » est « d'encourager la souscription des licences individuelles aux membres des associations de province et l'adhésion au C.G.P.I.F. pour les associations franciliennes » (Sprint Express, 11, 1998: 7). Les raisons évoquées de cette participation à des rencontres non-exclusivement gaies et/ou lesbiennes, sont que les homosexuels peuvent alors parvenir à une visibilité et acquérir " une reconnaissance et un respect de la communauté homosexuelle...», en leur offrant aussi la possibilité de participer à des tournois sportifs spécifiquement homosexuels mais " sanctionnés ", c'est-à-dire "justes, officiels et fun ", grâce aux règles valables pour tous et une reconnaissance officielle du niveau sportif par l'enregistrement des records visant encore à influencer positivement le regard de l'Autre sur la cause homosexuelle (Gouyet S., 1999). Cette appréciation est corroborée par des nageurs du club du Paris Aquatique, club dont l'autorité sportive est reconnue par le milieu officiel: "D'être reconnu comme un véritable club de natation, là se pose la question de la véritable homologation. Je pense qu'à long terme ce sera forcément l'homologation pour tous les clubs, pour tous les sports... C'est seul moyen, vraiment, de se faire reconnaitre ».

Il est aisé de comprendre que cette stratégie ouverte au sport homosexuel engendre des effets pervers, notamment vis-à-vis de la mixité de pratiques sportives comme la danse ou le patinage artistique qui entrent en contradiction avec les normes officielles. L'alternative devient simple, ou se soumettre ou se démettre. Ce fut le cas pour l'épreuve de patinage artistique à Amsterdam puisque l'I.S.U. (International Skating Union) fit savoir qu'elle refusait de considérer officiellement cette épreuve et que les juges et les patineurs qui y participeraient seraient exclus des futures épreuves qu'elle organiserait. Malgré les protestations, la compétition de "figures" fut annulée et transformée en entraînement public. Quelques patineurs portaient un masque sur le visage à cette occasion. De plus, des règlements spécifiques continuent d'être appliqués lors des jeux de Sydney dans l'épreuve de danse, où les couples formés peuvent être du même sexe, ou en danse avec fauteuil ou le handicapé fait sa prestation avec un partenaire valide, ou en tennis apprécié selon les règles de la G.T.L.A. (Gay and Lesbian Tennis Alliance) afin de permettre une séparation des âges et un accès aux personnes en fauteuil roulant.

Des réseaux ouverts aux notables

L'institutionnalisation de l'environnement concerne aussi le processus de recherche de transactions avec des notables des collectivités territoriales ou avec des groupes d'intérêts économiques sensibles au développement du sport en général. A priori éloignés de la défense de la cause homosexuelle ils répondent cependant favorablement - pour des raisons politiques ou financières - aux demandes exprimées par cette population spécifique : le système s'élargit alors à un réseau d'organisations politiques et économiques. Il s'agit ici, d'un mouvement de retour, ce pont dont le passage est emprunté par l'Autre pour aller vers le sport homosexuel, même si l'offensive vient du minoritaire. Ces transactions constituent des informations qui en pénétrant dans le système, bouleversent l'équilibre initial et attestent de son ouverture.

Ces alliances faites de reconnaissances réciproques prennent différentes formes de gratification repérables dans des actes de la vie quotidienne, comme par exemple la 
remise d'une Coupe de la ville de Paris par l'adjoint au Maire du XIème arrondissement, à la Présidente d'alors du C.G.P.I.F., pour la remercier de l'action de la Fédération menée en faveur du sport, au Président du Paris Aquatique pour l'organisation du Championnat mondial IGLA à Paris et au co-président actuel de C.G.P.I.F. pour son action associative. Ce sont là des événements-transactions dont les effets sur la Fédération Sportive Gaie et Lesbienne ne sont pas neutres. Ils expriment symboliquement le gage d'une reconnaissance officielle pour le développement du sport qui crédite une culture marginalisée, la renforce dans ses convictions et la justifie dans l'option stratégique choisie ${ }^{14}$.

81 De même l'appui de notables des municipalités permet la réalisation de grands événements sportifs gays et lesbiens et ceci dans des formes parfois insoupçonnées, comme ce fut le cas pour les Gay Games d'Amsterdam où la municipalité, portée par une tradition libérale à l'égard de l'homosexualité, s'est portée garante à hauteur de 5 millions de florins du déficit économique (déficit estimé entre 1 million et 1 million et de demi de florins, soit près de 7 millions d'euros) ${ }^{15}$, et dont les qualités d'accueil et de solidarité ont surpris les participants : la ville s'était mise aux couleurs homosexuelles, rose, jaune et arc-en-ciel et les policiers distribuaient des cartes de bienvenue, « Fiers d'être vos amis ».

De même l'économie s'ouvre à d'autres réseaux que les organisations spécifiquement gaies, comme K.L.M. ou le groupe Accor, pour établir des partenariats et rechercher des financements car les Gay Games sont de véritables entreprises économiques (12 500 dollars U.S en $1982 ; 350000$ en $19862 ; 2,1$ millions en $1990 ; 12$ millions en 1994 et des retombées de 400 millions pour la ville de New York).

Cette nécessité de recourir à des partenaires économiques pour alimenter l'essor du sport gay et lesbien est l'objet de débats sur l'éthique relativement au choix des sponsors et à l'image qu'ils véhiculent, notamment vis-à-vis du sexe, et qui doit être en adéquation avec le côté positif recherché par la pratique des sports. C'est ainsi que des discussions se sont engagées à la Fédération Sportive Gaie et Lesbienne C.G.P.I.F. sur une charte mise en place par l'équipe "Sponsoring » destinée à dégager les grands axes et les objectifs vis-à-vis de sponsors éventuels, dont il est précisé dans l'article $3:$ : La Fédération se refuse à démarcher toute société ou organisation ayant des activités commerciales à caractère pornographique et/ou impliquée dans le commerce sexuel de toute nature (bar) réseau...), sauf dans des situations présentant un caractère urgent et exceptionnel, et à l'exclusion des sponsors déjà agréés l'an passé». Deux visions étaient défendues, celle des " réalistes » et des " puristes » du non-sexe, sans qu'une alternative ne se dégage des échanges (le C.G.P.I.F ne pourrait exister sans la revue Gai Pied ce qui implique quelques probables concessions). Mais la question posée portait aussi sur la possibilité ou non de faire abstraction de la dimension sexuelle extra-sportive. Car le style de vie des homosexuels les amène à fréquenter des lieux de rencontres et des réseaux sexuels, une voie contraire à l'image ludique et fun qu'ils véhiculent et qui s'accorderait alors avec les préjugés hétérosexuels (les homosexuels sont des pervers). L'appel à l'économie nécessite-t-il la néantisation de la prise en compte de la spécificité sexuelle ? C'est bien ce type d'aporie et de conflits qui émerge de l'extension du sport homosexuel.

Des rituels de conformisation

84 Malgré ce qui a été mis en évidence dans la première partie de cet article, la production des techniques du corps lors des événements sportifs gays et lesbiens ne traduit pas seulement une désymbolisation de l'ordre sportif dominant envisagée dans la logique 
souverainiste. Le glissement progressif vers la compétition engendre des rituels présentant des analogies avec ceux qui régulent le sport hétérosexuel. Cela ne signifie pas la disparition de l'esprit fondateur de convivialité, ni celui de « l'être-ensemblehomosexuel», mais il se peut que cette orientation stratégique vers le pôle de la performance modifie la culture initiale et génère de nouveaux conflits au sein des acteurs du sport gay et lesbien. En effet, la reprise de l'ordre dominant se lit dans la communication du nom des vainqueurs, dans la comptabilité des médailles, dans la publication des records, dans le nombre de pratiquants, de nationalités, etc., même si l'inclusion de tous demeure inchangée. Ces métamorphoses sont recherchées ici dans deux indicateurs renversant les priorités : la visibilité homosexuelle qui ne devient plus essentielle et le lien social communautaire donnant de l'importance à l'identité sportive.

Une conversion sportive d'invisibilité

Les rituels sportifs de type militants ne sont pas partagés par l'ensemble des homosexuels. Certain(e)s préfèrent demeurer invisibles et ceci de deux manières : en s'inscrivant dans un club officiel pour taire leur homosexualité ("Si on n'adhère pas à Aquahomo, c'est à cause du nom. C'est trop affiché»), ou en adhérant à un club gay et lesbien sans faire valoir leur appartenance homosexuelle face aux autres sportifs. Des désaccords s'expriment alors entre les présidents de clubs et les responsables de Fédérations gaies et lesbiennes selon les attitudes de chacun : les " militants " pensent que la reconnaissance du niveau de performance est une occasion de transformer l'image négative, à condition que le coming out soit réalisé ; les « sportifs ", pensent que c'est avant tout le niveau de performance qui prévaut et il n'est pas important (pour les nouveaux clubs dont les participants sont déjà performants) ou plus nécessaire (pour des clubs anciens qui ont fait leur preuve) d'afficher son homosexualité avant d'avoir été reconnus comme sportifs. Ainsi, un ancien président du club de natation Paris Aquatique déclare : «La première fois qu'on a été aux Championnats de France, c'était dans la grande piscine de Paris, c'était un Master. On avait notre drapeau gay qui pendait pendant deux jours. Bon! Maintenant, ça suffit. Les grands clubs le savent ", et la présidente du Football Club Paris Arc-en-Ciel confie: "Dans nos statuts, rien n'évoque que nos équipes sont homosexuelles. Nous préférons nous intégrer avant de le dire. On est footeux avant tout (...). Nous avons même changé le nom du club pour nous prémunir d'éventuelles discriminations (anciennement Gays Go Girls). Des jeunes du quartier ou même des entraîneurs nous traitaient de "pédés" et "gouines", et nous ne voulions pas que les clubs contre lesquels nous pourrions jouer, nous mettent tout de suite une étiquette. Nous venons pour jouer au football avant de militer (...) Nous sommes des êtres humains avant d'être des homosexuels. Je suis une femme avant d'être lesbienne (...). Nous, on joue au foot et on veut s'intégrer dans les deux mondes $»^{16}$.

Cette priorité sportive par rapport au militantisme du coming out est une transformation qui affecte la dynamique du mouvement dans ce qu'il a de politique : « On est là pour faire la fête, pour faire du sport. Ce n'est pas un acte de militantisme, c'est un tournoi sportif "; " Moi, je pense que c'est important qu'une sportive de haut niveau affirme son homosexualité. S'il y avait plus de sportifs hommes et femmes qui le faisaient, je pense que ce serait bien. En même temps, je ne peux m'empêcher de penser que ce sont des histoires individuelles et qu'on doit respecter le silence des uns et des autres" ; "On ne demande pas aux sportifs de haut niveau s'ils sont homosexuels ou hétérosexuels, surtout hétérosexuels... On s'en fout... Ça n'apporte rien à la carrière sportive, et je pense que ça n'apporte rien à la communauté homosexuelle $»^{17}$. 
87 Ici s'affichent des priorités festives de convivialité et de compétition, sans qu'elles ne soient reliées à un acte militant dûment revendiqué comme tel, comme si la pratique de haut niveau dépolitisait le mouvement homosexuel et donnait raison à la thèse de la transfiguration du politique et à la puissance des émotions vécues dans le moment. Ces données qualitatives sont confirmées par une enquête menée auprès de 151 participants aux Euro Games de Paris en 1997 portant sur les raisons de leur engagement sportif (échantillon composé de 93,2\% d'homosexuels, de 5,4\% de bisexuels et 1,4\% d'hétérosexuels). Pour 90,6\% de la population interrogée, le sport apparaît comme un facteur d'intégration et les résultats attestent de la recherche de valeurs de convivialité (58,9\%), puis de compétition (58,3\%), faire des rencontres (53,6\%), défendre la visibilité homosexuelle (44,3\%), représenter un club $(29,7 \%)$, lutter contre l'homophobie $(21,4 \%)$, défendre les couleurs de son pays (9,9\%). Tout se passe comme si le mouvement sportif initié contre l'homophobie perdait de sa conviction militante avec l'élévation du niveau sportif. La vision politique collective de la défense de la cause homosexuelle (visibilité, lutte contre les discriminations) est moins prisée que les valeurs provenant de demandes individuelles que le collectif aide à réaliser. Ceci se confirme par les réponses à la question de savoir si le mouvement sportif homosexuel doit, ou bien faire partie complètement $d u$ sport international ou national officiel $(82,3 \%)$, ou bien être complètement autonome $(17,7 \%)$. Les taux de réponses ne sont pas significatifs selon le sexe, mais le deviennent avec la variable «niveau sportif» (débutant et initié/ confirmé). Le choix de l'affiliation avec le sport officiel croît avec l'élévation du niveau d'habileté physique estimé. La relativisation du caractère mobilisateur s'affirme encore dans les réponses à la question de savoir ce que les Euro Games suscitent chez les participants. Sont majorées les valeurs compétitives comme la participation aux Euro Games (81\%), aux Gay Games (79,7\%), renforcer son entraînement (66,3\%), plutôt que celles qui promeuvent la sociabilité (organiser des rencontres $27,8 \%$, ou créer une association $4,4 \%$ ).

Invisibilité, déstructuration des statuts, valorisation des habiletés physiques de hautniveau sont privilégiées par rapport à l'affichage de son identité gaie ou lesbienne et contribuent à une normalisation avec les valeurs du sport officiel en transformant un " être-ensemble-homosexuel », en «être-ensemble-sportif-homosexuel», dans lequel l'identité sportive est maximisée, sans que soit niée pour autant la dimension homosexuelle. Cela témoigne de la part des homosexuel(le)s, de l'intériorisation au plan de l'imaginaire, des attendus du mouvement sportif dominant et de réponses concrètes par des pratiques de conformisation.

Des rituels affinitaires

89 Enfin, l'observation des compétitions lors de Gay Games ou de Tournois européens et les témoignages de participant(e)s rendent compte de l'importance de la performance et de ce qu'elle nécessite comme entraînements. Cela se perçoit aussi dans l'intériorisation des gestuelles des pratiques sportives officielles, ce qui rapproche encore les deux mondes.

90 Ainsi, les vêtements, les apparences, les techniques du corps et les rituels observés lors d'un tournoi lesbien de volley-ball, ne diffèrent pas fondamentalement de ceux d'une rencontre du sport hétérosexuel. Des similitudes existent quant aux apparences significatives de l'appartenance au monde des sports (port de genouillères, musculature témoignant d'une pratique régulière, tenues homogènes, emblèmes $d u$ club), quant aux rituels de préparation au match (ordre des échauffements, passes, 
manchettes, smashes d'abord en souplesse puis plus tendus, déplacements latéraux, jeux d'attaque et de défense, ceci dans le seul bruit des pas, des frappes et des ballons), à ceux du match (accompagnement des points réussis par des claques d'encouragement dans les mains), ou encore à ceux de l'après-match (les équipes main dans la main saluent le public, avec cependant la particularité du baiser sur la bouche entre les joueuses). Les nageurs en natation synchronisée du Paris Aquatique ne peuvent prétendre à leur réussite sans la rigueur de trois entraînements par semaine sous la direction d'une spécialiste et les tableaux de performance en athlétisme ou en natation traduisent l'excellent niveau de certains participants. Bref, la logique sportive en acte signifie qu'une "performativité » comprise comme la recherche exacerbée de la performance caractéristique du sport officiel, (Le Pogam Y., 1995) n'est plus étrangère au mouvement sportif homosexuel.

91 Ainsi, d'autres formes de métissage se mettent en place, non plus seulement dans l'emprunt des techniques sportives orthodoxes, mais dans la production d'analogies avec les valeurs du sport officiel, tant dans les idéologies (appel au système axiologique de l'intégration par le sport comme pont entre les cultures), que dans les structures organisationnelles (recherche d'isomorphismes entre les organisations gaies ou lesbiennes et officielles, homologation des clubs, échanges avec les notables de la politique du sport), que dans des rituels signifiant l'effacement symbolique de la préférence sexuelle dans l'objectif d'atteindre une excellence sportive débarrassée d'une forme de rébellion. Ce deuxième pôle stratégique d'agrégation ou de normalisation, n'apparait plus comme un contre-pouvoir issu d'une contre-culture visà-vis de l'ordre symbolique dominant. Cette stratégie de ressemblance, s'opposant à la logique de dissemblance revendicative et militante pourrait a priori être interprétée comme étant contre-productive dans la réalité et dans l'imaginaire. Elle génèrerait un effet pervers entraînant la relativisation des valeurs transgressives et un renoncement à la logique souveraine, constitutive de l'émergence du mouvement sportif homosexuel.

Mais peut-être faut-il dépasser cette apparente contradiction. Le dominé peut ne pas perdre son identité en subissant une normalisation qui demeure partielle, grâce à une ruse avec la domination. Il laisse croire que l'adoption de la règle officielle le rend dépendant, alors qu'il ne renonce pas pour autant à sa préférence sexuelle. Ici, le jeu reste dans le domaine de la représentation, de l'image présentée, du signifiant et de l'expression, alors que le signifié demeure inchangé. L'acceptation peut être alors considérée comme une charge symbolique très forte : la ruse de l'apparence peut être une stratégie du " comme si » (nous épousions les valeurs dominantes), sans céder une parcelle de son identité fondatrice. Alors, le pouvoir est du côté du dominé, en ce sens que le dominant, en croyant avoir soumis l'Autre à la norme, finit par le reconnaître dans sa différence, dans son existence, sans penser que l'Autre peut générer la propre transformation de son éthos. 


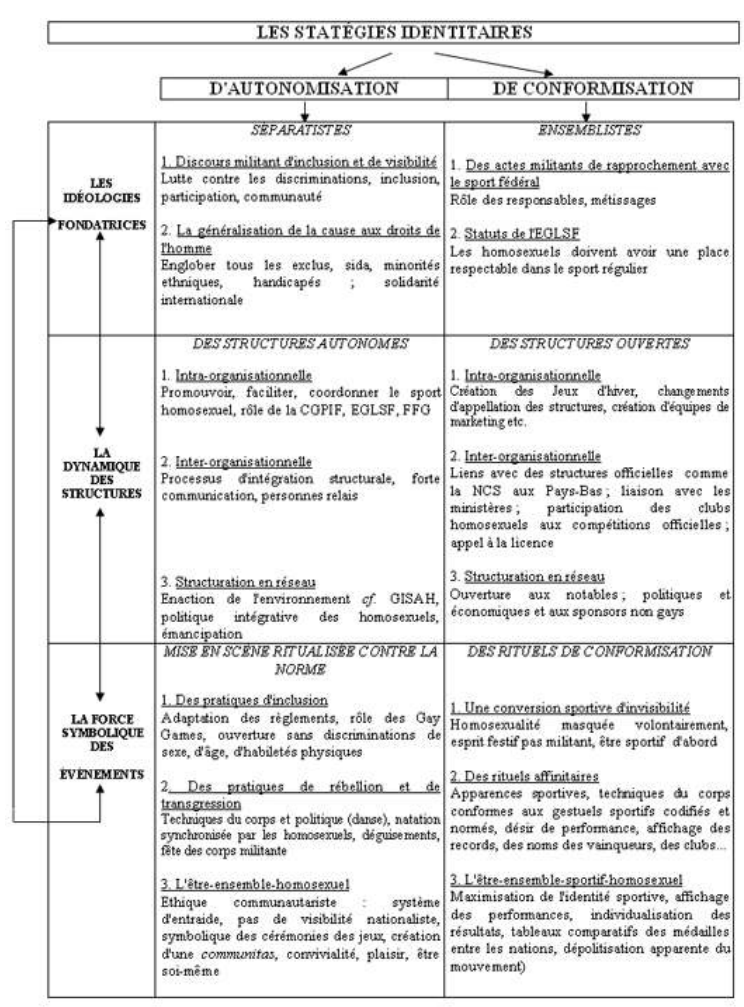

Le tableau doit être lu non pas dans sa linéarité (verticale ou transversale), mais bien comme un système dans lequel les dimensions, composantes et indicateurs sont dans une connexité qui entretient la dynamique du mouvement et engendre sa transformation par les conflits qui émergent.

Communauté et universalité des droits stratégies à la fois contradictoires et complémentaires qui ont en commun de viser les mêmes finalités: la légitimation de la cause homosexuelle par l'image positive véhiculée par le sport et la transformation des codes culturels dominants en œuvre dans la société ? Comment interpréter la spécificité du mouvement sportif gay et lesbien en regard des recherches sur les différences culturelles et sur les mouvements sociaux?

Institutionnalisation et éthique conflictuelle

ne sociologie attentive aux différences culturelles mentionne qu'elles ne sont pas socialement neutres mais qu'elles sont l'objet de conflits et procèdent de demandes mettant en cause la domination subie et l'exclusion vécue dans le cadre de la vie quotidienne, révélant ainsi une privation qui peut être économique ou symbolique (Wieviorka M., 1996). La structuration du mouvement sportif homosexuel vise bien à établir un contre-pouvoir d'ordre symbolique visant une égalité de droits en regard d'institutions discriminantes structurées par l'ordre hétérosexuel. Il s'agit d'une confrontation, car des dominations se manifestent dans les usages sociaux du corps, ce qui est paradoxal dans des sociétés qui, comme le souligne Georges Balandier, se font de plus en plus «communicantes» (1994). Effectivement, les cultures homosexuelle et hétérosexuelle ne sont pas juxtaposées et leurs relations sont conflictuelles dans la mesure où persistent des inégalités, des rejets, des discriminations engendrant la négation de la personne et des menaces sur son identité. Cette question ne relève pas seulement d'une éthique, de la seule exigence de vivre-ensemble dans le respect des

Corps et culture, Numéro 6/7 | 2004 
différences. Ce serait se priver d'une réalité sociologique et politique qui implique la lutte comme contre-pouvoir à l'adresse de l'ordre symbolique surplombant et la mise en place d'un répertoire d'actions collectives. Ce répertoire coordonne les deux stratégies, évitant les extrêmes que seraient d'un côté une vision intégriste, séparatiste et enfermante et de l'autre, une vision assimilationniste, confondue avec la culture dominante de l'institution sportive légitimée.

La dynamique instituante du sport homosexuel saisie dans ces deux stratégies organiquement liées entre elles, montre comment les idéologies proclamées par ceux qui produisent le mouvement, apparaissent comme des forces cognitives et motivantes pour une population stigmatisée afin de lier deux ambitions. La première consiste à produire des images identifiantes d'une communauté morale consensuelle réunie autour de la défense d'une cause légitime dans un Etat de droit. La seconde est attentive à ne pas s'enfermer dans un ghetto en s'ouvrant aux pratiques sportives reconnues par les instances fédérales officielles.

97 Cette puissance des idéologies politiques comme forces persuasives portées par un militantisme s'accompagnent de moyens organisationnels (structuration du mouvement, statuts, moyens d'adhésion), eux-mêmes orientés tantôt vers un renforcement identitaire où priment des relations « entre-soi », tantôt vers le système axiologique dominant des institutions sportives officielles. Enfin, idéologies et structures font système avec les mises en scène des pratiques corporelles où les rituels sont marqués à la fois par l'emprise symbolique communautariste et par l'adhésion aux codes et aux gestuelles conformes à la logique de la performance. Mais ce que montre l'union de ces deux stratégies contradictoires, c'est aussi la présence de conflits internes au mouvement sportif homosexuel lui-même, générés par les sportifs confirmés qui font passer au second plan leur spécificité homosexuelle (invisibilisation), avec la recherche de sponsors (image sexuellement lisse de l'homosexualité s'accordant avec les représentations du sport), et la déstructuration des techniques sportives transgressives (démixage des pratiques en accord avec les règlements officiels), ce qui rend la revendication militante moins agressive.

Ces transformations inédites cohabitent avec leur contraire au sein du mouvement gay et lesbien. Elles montrent que la décolonisation du pouvoir hétérosexuel par le sport est un processus incessant et inachevé marqué par des contradictions entre une logique de démarquage de la culture sportive hétérosexuelle et une logique d'emprise et de reprise officielle re-masculinisante.

Mais le sport hétérosexuel peut être affecté en retour. Ainsi, par les déconstructions sexuées opérées par les rituels festifs et rebelles (notamment par l'inversion des séparations catégorielles du masculin/féminin dans le cas de la natation synchronisée spécifiquement féminine dans le sport officiel par exemple), le sport gay et lesbien montre au sport officiel qu'il est construit sur une logique à la fois sexiste, sexuelle et genrée et qu'il n'est pas démocratique malgré ses idéologies proclamées dans la mesure où il crée socialement des inégalités de droits et de faits. Comme tel, ce dévoilement peut l'engager à des transformations pour remédier à ces failles ${ }^{18}$. Alors, l'éthique conflictuelle à l'égard de l'hétérosexualité ne transforme pas seulement la culture d'origine qui se virilise pour les plus performants, mais encore la culture dominante par un effet de retour, en exhumant les fondements inconscients qui la structure et en déstabilisant les signifiés sport/genre-sexe-sexualité qu'elle institue.

La portée alternative du mouvement sportif gay et lesbien 
100 L'exemple du sport gay et lesbien montre que la virilité des corps n'est pas antinomique avec le caractère efféminé pour jouer en se jouant du dominant : les corps des nageurs gays du Paris Aquatique sont des corps sportifs sculptés par les nombreux entraînements, cela ne les empêche pas de jouer avec la féminité par la pratique de la natation synchronisée comme dévoilement de ce qu'est l'ordre masculin dans le système sportif officiel. La subversion ne relève pas alors d'une symbolique "pure ", mais se présente comme une alternative communautariste qui peut affecter en retour le basculement, du moins partiel, de l'androcentrisme des institutions sportives dominantes et agir ainsi dans le cadre d'une problématique plus universaliste de la différence instituée entre les genres (ce qui serait le cas dans un scénario qui n'est pas inimaginable sur une longue durée, où les hommes - homosexuels ou/et hétérosexuels -, auraient toute légitimité à pratiquer la natation synchronisée dans les institutions officielles qui l'auraient admise, comme les femmes ont eu accès à des pratiques jugées masculines, ce qui ne signifie pas pour autant la fin des inégalités).

De même, le répertoire d'actions collectives de la stratégie de normalisation du sport gay et lesbien dépasse les seules revendications contre l'homophobie, pour les généraliser à toutes les formes d'exclusion, quelle que soit l'origine du stigmate (ethnies, handicapés, sidéens), en sensibilisant les Etats-Nations, les Ministères et les organisations à la défense des droits de la personne. Mary Bernstein (1997 : 532), dans sa recherche sur l'usage stratégique de l'identité dans le mouvement gay et lesbien, distingue bien les droits de l'homme visant à protéger des discriminations dans le logement, l'emploi, les relations publiques basées sur des caractéristiques comme le sexe, la race, les origines nationales, des droits des gays et des lesbiennes qui concernent les orientations sexuelles rapportées aux bases précédentes. En cela, les revendications, bien que sexuellement déterminées, produisent des effets généralisables aux autres exclus, comme l'accès de tous les sports ou l'obtention du PaCS (le pacte civil de solidarité avec 56000 contrats souscrits dont 60\% d'hétérosexuels), puisqu'il ne se rapporte pas exclusivement à leur cause mais affecte toutes les personnes vivant en couple, indépendamment de leur préférence sexuelle. En cela, les actions menées par les acteurs du sport homosexuel, participent d'une vision modernisatrice de la société fondée sur une volonté égalitariste, dont les effets, orchestrés, certes, au départ par une défense communautariste, peuvent «changer le monde » (slogan des Gay Games), parce que l'éthique sociale est construite sur une généralisation de la cause et concerne une politique universaliste des droits de l'homme.

102 Rapportés à la spécificité du mouvement sportif gay et lesbien, ces résultats montrent, par rapport à l'analyse de Mary Bernstein (1997), que la stratégie critique existe à l'égard des pouvoirs publics (par les revendications auprès des instances politiques, par la rébellion et la transgression des rituels, par l'organisation d'événements spécifiques mondialisés) et se poursuit conjointement avec la stratégie éducative (de normalisation et de passages vers le sport officiel). Les deux logiques sont connexes, indépendamment du degré de structuration des organisations et de l'accès à la politique et de l'opposition, puisque les stratégies de provocation ne cessent pas pour autant. Ici, la variable niveau sportif semble discriminante, puisque même si la provocation et la rébellion se retrouvent dans le sport récréatif et de performance, elles apparaissent d'autant moins fortes que le niveau sportif est élevé, c'est-à-dire quand les jeux se transforment en sport. Dans le sport gay et lesbien, l'usage stratégique de l'identité 
éducative supplée l'usage critique quand les structures organisationnelles et les acteurs se rapprochent du sport officiel et quand les pratiquant(e)s, grâce au niveau sportif atteint, accèdent à une reconnaissance des institutions légitimantes.

Cette recherche montre aussi l'usage de différentes formes stratégiques de la critique et leurs effets, quant à leur possibilité à produire des alternatives, ou des réformes sociales. La charge subversive des rituels du corps lors d'événements sportifs a-t-elle la même portée que la lutte menée contre les discriminations auprès des Institutions européennes, comme le Conseil de l'Europe par exemple? A priori, le mode critique indirect des premières actions collectives paraît moins sérieux que le second type d'actions, plus directement politiques. Mais pourtant, les rituels du corps atteignent bien les valeurs constitutives de la modernité en dévoilant, par leur dérision, les fondements axiologiques de la culture qui la caractérisent. Ils témoignent de l'existence d'un système dionysiaque (raillerie, féminisation des hommes dans leur apparence sportive, rébellion, transgression) à l'intérieur d'une culture dominante apollinienne constitutive du sport (ordre, mesure, maîtrise, rendement, performance, hiérarchies). En cela, le mouvement sportif homosexuel participe d'une dimension « expressive » du mouvement social selon la formule d'Alain Touraine (1996), au sens où il est un contrepoint à l'utilitarisme, aux normes de la rationalité calculatrice et quantitative de l'économie moderne, et ici, aux normes conquérantes et masculines, en montrant que des valeurs "féminines » peuvent être réintroduites dans l'ordre masculin, malgré une présence de la virilité. Ces rituels de provocation introduisent du désordre dans l'ordre institué et deviennent un analyseur symbolique critique qui attire l'attention sur l'importance des signifiés et non des signifiants. Le sport, alors, n'est plus qu'un jeu privilégiant la non puissance, construit contre la progression rationaliste, la discipline et l'intégration. En cela, cette forme de violence moins spectaculaire que les actions collectives menées directement auprès des institutions politiques n'en est pas moins efficace, parce que le corps, dans la mise en scène du jeu sportif, devient le mode d'expression des injustices et des désirs de réparation. La recherche exacerbée de l'excellence sportive, en ce qu'elle minimise ce pouvoir critique de la transgression pour se circonscrire à des rituels festifs vécus ici et maintenant, opère un changement d'orientation stratégique: la contestation ruse avec le pouvoir du dominant, en substituant la séduction à la provocation.

Le mouvement sportif homosexuel par les idéologies promues et les pratiques divulguées, permet de joindre le procès de subjectivisation par la valorisation des différences, l'affirmation identitaire, le communautarisme, une dépolitisation des forces militantes, l'esprit ludique, la fête, au procès de rationalisation constituée par la défense de principes universels tels que les droits de la personne, ou même les lois de l'économie et du marché nécessaires à l'édification des jeux. L'hypothèse d'un retour au sujet (Touraine A., 1992, 1994 ; Wieviorka M., 2000) a ici toute sa pertinence dans la mesure où les questions d'éthique et d'estime de soi ne s'appliquent pas aux seuls critères d'une préférence sexuelle. Cette institutionnalisation basée sur un travail de reconnaissance s'affirme comme réaction au pouvoir dominant en s'édifiant sur la puissance des affects où il est question d'expériences vécues, de sentiments, d'émotions, de mémoire, de plaisir d'être ensemble, de combat juste, de recherche de sens dans les actions collectives. Ce sont là des valeurs dégagées d'une conception utilitariste exclusive, sans qu'elles soient cependant coupées d'une rationalité, qui, articulée sur une universalité des droits ou des logiques économiques, vise à ce que se concrétise par les jeux sportifs, l'utopie de vouloir «changer le monde». Une utopie 
qui ne peut conduire cependant à une totalité sociale enfin réconciliée, achevée et close.

\section{BIBLIOGRAPHIE}

Ansart P. (1979) Eléments d'épistémologie pour une sociologie des affects politiques, Université Paris VII, Département de sociologie, ronéotypé, non publié, $110 \mathrm{p}$.

Ansart P. (1984) L'imaginaire social, Encyclopædia Universalis, supplément Les Enjeux, 710-713.

Ansart P. (1988) L'imprévu et l'inédit en politique, Revue de l'Institut de Sociologie, Les nouveaux enjeux de l'anthropologie, Autour de Georges Balandier, 3-4, 1988, 191-200.

Augé M. (1994) Pour une anthropologie des mondes contemporains , Paris, Aubier.

Ataman K., Brooks P. (1994) Talons et pointes, Final Touch/Planète, 27 octobre.

Baillette F., Liotard P. (1999) Sport et virilisme, Montpellier, Ed. Quasimodo et fils.

Balandier G. (1994) Le Dédale. Pour en finir avec le XXè siècle, Paris, Fayard.

Balandier G. (2001) Le Grand Système, Paris, Fayard.

Barel Y. (1984) La Société du vide, Paris, Ed. du Seuil.

Bernstein M. (1997) Celebration and Suppression : The Strategic Uses of Identity by the Lesbian and Gay Movement, American Journal of Sociology, vol 103, 3, 531-565.

Chamberland L. (1997) Du fléau au fait social. L'étude des homosexualités, Sociologie et sociétés, Homosexualités : enjeux scientifiques et militants, 29, 1, 5-20.

de Lauretis T. (1991) Queer Theory : Lesbian and Gay Sexualities (An Introduction), Differences, A Journal of Feminist Cultural Studies, 3, 3-18.

Denis D. (1997) La Revanche des dominés, Quasimodo, Nationalismes sportifs, printemps, 3-4, 47-60.

E.G.S.L.F. (1999) European document of gay and lesbian discrimination in sport, Den Haag, The Netherlands.

E.G.S.L.F., G.I.S.A.H. (1999) Building bridges between regular and gay/lesbian sport, The Hague, The Netherlands.

Eng H. (1997) Queer studies and sport, paper presented at I.S.S.A. (International Sociology of Sport Association), Symposium Oslo, 28 juin-1er juillet.

Falcetta L. (1997) L'Emergence du mouvement sportif homosexuel, Mémoire de Maîtrise S.T.A.P.S., (dir. Y. Le Pogam), Faculté des Sciences du Sport, Université Montpellier1.

Good as you (2002) Va y avoir du sport, réalisation D. Tahi, création/production J. Caza, Canal Jimmy, 21 avril.

Gouyet S. (1999) Le sport homosexuel en France, http://perso.wanadoo.fr/cgif/reseau/ français:conferencereport.htm. 
Griffin P. (1992) Changing the Game : Homophobia, Sexism and Lesbians in Sport, Quest, 44, 251-265.

Gurvitch G. (1950) La Vocation actuelle de la sociologie, Paris, P.U.F.

Hekma G. (1994) Als ze maar niet provoceren. Discriminatie van homoseksuele mannen en lesbische vrouwen in the sport, Amsterdam, Het Spinhuis.

Hekma G. (1997) Les limites de la révolution sexuelle. Grammaire de la culture sexuelle occidentale contemporaine, Sociologie et Sociétés, Homosexualités : enjeux scientifiques et militants, 29, 1, 145-156.

Kolnes J.-J. (1995) Heterosexuality as an organizing principe of women's sport, International review for the sociology of sport, 30, 1, 61-80.

Laberge S. (1995) Sport et activités physiques : modes d'aliénation et pratiques émancipatoires, Sociologie et Sociétés, (Le Sport), 27, 1, 53-74.

Lefevre N. (1998) Les Euro Games V : un analyseur du réseau sportif gai et lesbien, Corps et Culture, (Sport et lien social), 3, 57-67.

Le Pogam Y (1995) Imaginaire sportif et fantasme de la performativité, Cahiers internationaux de Sociologie, Force et contagion des émotions, vol XCVIII, 23-41.

Le Pogam Y. (1997) Passions sportives, identité et modernité, Quasimodo, Nationalismes sportifs, printemps, 33-46.

Le Pogam Y (1998) Michel Maffesoli, analyste de la socialité émergente, Corps et Culture, (Sport et lien social), 3, 203-229.

Le Pogam Y. (1999a) Sport, modernité et souveraineté, Les Cahiers de l'Imaginaire, Arts de vivre, Université Paul Valéry, Montpellier, 95-108.

Le Pogam Y. (1999b) Rites du sport et générativité du social, Corps et Culture, (Corps, sports et rites), 4, 147-175.

Liotard P. (2003), Sport, in Tin J.-L. (sous la direction de), Dictionnaire de l'homophobie, Paris, PUF, 383-386.

Maffesoli M. (1982) L'Ombre de Dionysos, Contribution à une sociologie de l'orgie, Paris, Librairie Méridiens/Anthropos, Rééd. (1991), Le Livre de Poche, 4131.

Maffesoli M. (1988) Le Temps des tribus, Le déclin de l'individualisme dans les sociétés de masse, Paris, Méridiens-Klincksieck, Rééd.(1991), Le Livre de Poche, 4142.

Maffesoli M. (1990) Au creux des apparences, Pour une éthique de l'esthétique, Paris, Plon, Rééd. (1995), Le Livre de Poche, 4223.

Maffesoli M. (1992) La Transfiguration du politique, La tribalisation du monde, Paris, Grasset/ Frasquelle, Rééd. (1995), Le Livre de Poche, Biblio Essais.

Marcellini A., Lefevre N., de Léséleuc E., Bui-Xuân G. (2000) D'une minorité, l'autre... Pratiques sportives, visibilité et intégration sociale de groupes stigmatisés, Loisir et Société/Society and Leisure, 23, 1, 251-272.

Mauss M. (1924) Essai sur le don, in Sociologie et anthropologie, Paris, Ed. Universitaires (1968).

Messner M.A, Sabo D.F. (Eds) (1994) Sex, violence and power in sports : rethinking masculinity, Boston, Beacon Press.

Morin E. (1990) Introduction à la pensée complexe, Paris, ESF Editeur. 
Neveu E. (1996) Sociologie des mouvements sociaux, Paris, Ed. La Découverte.

Pouliquen G. (2002) L'Homophobie dans le sport, Mémoire de D.E.A (Dir. Xiberras M., Liotard P.), Université Montpellier III, $74 \mathrm{p}$.

Pronger B. (1990) The Arena of masculinity, Sports, homosexuality, and the meaning of sex, New-York, St Martin's Press

Rotella R.J., Murray M.M. (1991) Homophobia, the world of sports, and sport psychology consulting, The Sport Psychologist, Vol 5, 355-364.

Saouter A. (2000) Être rugby, Jeux du masculin et du féminin, Paris, Maison des Sciences de l'Homme. Sansot P. (1986) Les Formes sensibles de la vie sociale, Paris, P.U.F.

Schuyf J., Stoepler L. (1998) Sexuality unknow, (abstract), gaysport.org/gisah/survey 2.

Stein A., Plummer K. (1996) I can't even think straight, in Seidman S. (Ed.), Queer theory sociology, Cambridge, Blackwell Publishers Ltd.

Tilly F. (1986) La France conteste de 1600 à nos jours, Paris, Fayard.

Touraine A. (1985) La société a-t-elle un centre ?, in Maffesoli M., Rivière C. (sous la direction de), Une anthropologie des turbulences, Hommage à Georges Balandier, Berg International Editeurs, 99-115.

Touraine A. (1992) Critique de la modernité, Paris, Fayard, Biblio Essais, 4217.

Touraine A. (1994) Qu'est ce que la démocratie ?, Paris, Fayard.

Touraine A. (1996) Faux et vrais problèmes, in Wieviorka (sous la direction de), Une société fragmentée ? Le multiculturalisme en débat, Paris, Ed. La Découverte, 291-319.

Turner V.W. (1969) Le Phénomène rituel, structure et contre-structure, Paris, P.U.F. (1990).

Wieviorka M. (1996) Culture, société, démocratie, in Wieviorka (sous la direction de), Une société fragmentée ? Le multiculturalisme en débat, Paris, Ed. La Découverte, 11-60.

Wieviorka M. (2000) Sociologie postclassique ou déclin de la sociologie ?, Cahiers internationaux de Sociologie, (Sociologies inactuelles, sociologie actuelles ?), vol CVIII, 5-35.

\section{NOTES}

1. Dans un article de synthèse des recherches gaies et lesbiennes depuis le années 70 dans les pays anglo-saxons et en France (n'incluant pas celles qui se rapportent au sport), Line Chamberland fait émerger d'un point de vue diachronique l'institutionnalisation universitaire des travaux et l'épistémologie qui les sous-tendent en discriminant cinq paradigmes : existentialiste, essentialiste, constructiviste, critique de l'ethnocentrisme et enfin depuis le milieu des années 80, des théories Queer postféministes et post-modernes opposées aux théories constructivistes ou essentialistes avec des méthodes issues de la sémiologie et du déconstructionnisme. L'auteure montre bien que les « enjeux et les débats actuels » sur les Etudes Gaies et Lesbiennes et les Etudes Queer, concernent moins les thèmes que les postulats théoriques qui les interrogent, les théories Queer sont l'objet de critiques car elles sont spécialisées en études littéraires peu ouvertes aux théories anthroposociologiques (Line Chamberland L., « Du fléau au fait social. L'étude des homosexualités ", Sociologie et sociétés, Homosexualités : enjeux scientifiques et militants, 29, 1, 5-20, 1997). 
2. Le recours aux appellations « " gay » et « lesbienne » répond à la volonté de prendre en compte la spécificité des problématiques homosexuelles en fonction du genre. 3. Dans le texte, une distinction doit être faite à propos de l'usage de la notion de « mouvement ». L'idée de « mouvement sportif homosexuel » renvoie ici, classiquement, à l'ensemble des acteurs concernés par ce phénomène (membres des Fédérations, des organisations, sportifs) acceptant d'être dirigés par l'idéologie fondatrice. Cette définition académique n'engage aucun présupposé théorique pour comprendre la dynamique des actions collectives, ce qui la différencie du « mouvement social » qui se rapporte à un paradigme « actionnaliste » (ou « actionniste » selon les auteurs), bien identifié et théorisé dans les sciences sociales et qui sert de support ici à la théorisation à la recherche.

4. La violence envers les homosexuel(le)s dans le sport n'est pas toujours symbolique.

Cf. Rotella and Murray (1991)

5. Afin de légitimer leur mouvement, les organisateurs du sport homosexuel ne manquent pas de souligner que ce n'est que vers les années 70 que certains sportifs prestigieux osent avouer leur homosexualité, comme Billie Jean King ou Martina Navratilova, ou plus récemment Amélie Mauresmo ou le nageur Greg Louganis, médaille d'or aux Jeux Olympiques de Los Angeles. Il semble, malgré le temps, que les attitudes à cet égard ne soient pas univoques. Ainsi, la notoriété de Ian Roberts, un rugbyman australien prestigieux, n'a pas été affectée après son coming out, mais Justin Funashu, un footballeur anglais, s'est donné la mort juste au début de la Coupe du Monde en 1998. Espoir dans les années 80, il était devenu le bouc émissaire de la profession, se faisait traiter de « «sale tante " par son entraîneur et il ne pourra surmonter ces longues années d'humiliation. Cela ne saurait non plus occulter les pressions exercées par les sponsors dont a souffert par exemple le nageur canadien Marc Tewsburg, qui a perdu des contrats en 1998 pour cette raison.

6. Cela est confirmé lors de nos entretiens : « Le sport est une valeur qui n'appartient à personne. Alors pourquoi sport et homosexualité ? Tout simplement parce que les homos sont des gens qui très souvent dans leur jeunesse ont vécu le sport comme une humiliation, puisque le sport est l'expression de valeurs masculines. Quelques années plus tard, quand ils s'aperçoivent $q u$ 'il ont des muscles et un corps et qu'ils veulent rendre leur corps désirable, le sport est le seul moyen pour eux de redonner toute la valeur corporelle ".

7. Aux Gay Games d'Amsterdam en 1998, les femmes pratiquent majoritairement les sports collectifs, et les hommes, les sports individuels (natation, body building, athlétisme), excepté le volley-ball.

8. Ce que semblent confirmer les premiers résultats d'une étude comparative menée par notre groupe de recherches entre trois pays distingués par les critères d'ancienneté du sport homosexuel et de libéralisme politique à leur égard : les Pays-Bas, La France et l'Espagne.

9. La notion de "stratégie agrégative », renvoie dans le texte à la définition académique "d'assemblage dans un tout », ici les actions menées par le sport homosexuel pour s'unir au sport officiel (être ensemble), et non au paradigme sociologique de l'individualisme méthodologique tel qu'il a été développé par Raymond Boudon (Effets pervers et ordre social, Paris, 1977 ; La Place du désordre, Paris, PUF, 1984) et Jean G. Padioleau (L'Ordre social, Paris, L'Harmattan, 1986), dans lequel les phénomènes sociaux s'expliquent par l'agrégation d'actions individuelles non recherchées par les agents d'un système, résultant de leur interdépendance. 
10. Un approfondissement de cette analyse est prévu à partir d'une observation participante des Euro Games de Copenhague en 2003.

11. Cette question de la révélation du statut H.I.V. dans le sport a fait l'objet de longues discussions au sein de la Fédération Sportive Gaie et Lesbienne C.G.P.I.F. Sollicitée par le magazine gai Têtu préparant un guide sur le H.I.V., elle n'a pas voulu le renseigner sur ce très délicat sujet qui relève de l'intimité de la personne en argumentant que c'est là un rôle tenu par d'autres associations que la leur. En effet, le coming out dans le sport est loin d'être effectif et la révélation de la séroposivité demeure exceptionnelle (cas de Magic Johnson qui n'a d'ailleurs rien perdu de sa notoriété). Mais cela n'empêche pas la Fédération d'engager une mobilisation politique au sujet des risques liés au sport ou à prévention dans le milieu compétitif, en visant à sensibiliser les Ministères de la Santé, des Sports, le Comité National Olympique ou les associations, ni de développer une réflexion sur le rôle d'une pratique sportive régulière préconisée par de nombreux médecins pour compenser les effets secondaires des trithérapies. (cf. Christophe Claudel, Sprint Express, 16, 1999 : 16).

12. Tom Waddell avait créé pour cela le San Francisco Arts \& Athletics (S.F.A.A.), mais suite à une décision de la Cour Suprême des Etats-Unis en 1987, la notion d'Olympisme a dû être abandonnée.

13.. Dans le texte, la notion de « sport officiel » a été préférée à celle de « sport régulier ", afin de marquer son autorité institutionnelle et sa légitimité.

14. Ces alliances réciproques structurent la vie associative du sport homosexuel quand par exemple, la Mairie du XIXème arrondissement de Paris autorise la tenue des Assemblées Générales de la Fédération Gaie et Lesbienne C.G.P.I.F. (parfois longues de 7 heures), dans la salle de mariage et il est d'usage, qu'en la circonstance, le Maire dans son allocution souhaite la bienvenue à la Fédération.

15. Ce qui a entraîné d'ailleurs le limogeage du directeur des Jeux qui avait dépassé le budget sans en discuter avec les autres responsables. La démarche de la municipalité d'Amsterdam a permis aux jeux de se poursuivre pour la première fois dans une capitale européenne.

16. Cette affaire a fait l'objet d'une mise au point de la part du Président du C.G.P.I.F., car le club avant d'obtenir son adhésion avait été mis à l'essai, conformément aux statuts du C.G.P.I.F. Sa Présidente pensait qu'il s'agissait d'une exclusion liée à sa position. Ce malentendu, cependant significatif de transformations, s'est conclu par l'adhésion sans réserves du club à la Fédération.

17. Cet aspect très subjectif du coming out est bien exprimé par Amélie Mauresmo qui déclarait : « On peut se fragiliser en se mettant en avant comme je l'ai fait en Australie en 1999 quand j'ai annoncé que j'étais homo, c'est que dans un premier temps ça m'a fragilisée, j'en suis consciente, mais j'en suis sortie encore plus forte. Sur le long terme, quand je fais le bilan, je le répète, j'en suis sortie beaucoup plus forte. Mais c'est certain que ça ne s'est pas fait du jour au lendemain. » L'Equipe, 26/08/2002.

18. L'histoire du sport est marquée par la reprise de mouvements contestataires au sein du sport officiel, qu'il s'agisse des revendications des femmes, des pratiquants des sports de glisse ou des coureurs sur routes, toutes nées d'une volonté de rupture par rapport à la logique fédérale. En se structurant, ces mouvements se sont retrouvés encadrés par la logique officielle, signe que l'ordre dominant sait intégrer le désordre, même si son ordre initial est bouleversé, lui aussi, après ces opérations (cf. Yves Le Pogam, Rites du sport et générativité du social, Corps et Culture, «Corps, sports et rites », 4, 147-175). 


\section{RÉSUMÉS}

Les Etudes Gaies et Lesbiennes sont florissantes depuis les années 80, mais peu d'entre elles recourent à la notion de mouvement social pour analyser les actions collectives des communautés homosexuelles afin de revendiquer leurs droits. Par ailleurs, aucune de ces recherches n'a pris le sport homosexuel comme objet. Ici, deux stratégies sont étudiées dans le mouvement sportif gay et lesbien: une stratégie autonome, centrée sur l'identité et la communauté, et une stratégie agrégative orientée vers les institutions sportives officielles. Ces stratégies paradoxales et complémentaires génèrent des conflits internes et externes. Les résultats sont discutés par rapport aux recherches gaies et lesbiennes relatives au mouvement social.

Gay and Lesbian Studies are flourishing since the 1980s, but few of them take the notion of social movement to analyse collective behavior of homosexual communauties to insist on one's rights. In this way, any of these researches have taken homosexual sport as object. Here, are distinguished two strategies in gay and lesbian sport movement : a self-governement strategy centered on identity and communauty, and an agregative strategy oriented toward official sport institutions. These paradoxical and complementary strategies generate internal and external conflicts. The results are discussed in relation of gay and lesbian researches about social movement.

\section{INDEX}

Mots-clés : homophobie, homosexualité, identité, mouvement social, stratégie

Keywords : homophobia, homosexuality, identity, social movement, sport, strategy

\section{AUTEURS}

\section{YVES LE POGAM}

Equipe Corps et Culture, Faculté des Sciences du Sport, Université Montpellier I

\section{PHILIPPE LIOTARD}

C.R.I.S (Centre de Recherche et d'Innovation sur le Sport), Université Lyon 1.

\section{SYLVAIN FEREZ}

Equipe Corps et Culture, Faculté des Sciences du Sport, Université Montpellier I

\section{JEAN-BERNARD MARIE MOLES}

Equipe Corps et Culture, Faculté des Sciences du Sport, Université Montpellier I

\section{GUILLEMETTE POULIQUEN}

C.R.I.S (Centre de Recherche et d'Innovation sur le Sport), Université Lyon 1. 